\title{
Circular RNA: metabolism, functions and interactions with proteins
}

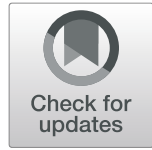

Wei-Yi Zhou ${ }^{1 \dagger}$, Ze-Rong Cai ${ }^{1 \dagger}$, Jia Liu ${ }^{1 \dagger}$, De-Shen Wang ${ }^{1}$, Huai-Qiang Ju ${ }^{1,2^{*}}$ and Rui-Hua $\mathrm{Xu}^{1,2^{*}}$ (D)

\begin{abstract}
Circular RNAs (CircRNAs) are single-stranded, covalently closed RNA molecules that are ubiquitous across species ranging from viruses to mammals. Important advances have been made in the biogenesis, regulation, localization, degradation and modification of circRNAs. CircRNAs exert biological functions by acting as transcriptional regulators, microRNA (miR) sponges and protein templates. Moreover, emerging evidence has revealed that a group of circRNAs can serve as protein decoys, scaffolds and recruiters. However, the existing research on circRNAprotein interactions is quite limited. Hence, in this review, we briefly summarize recent progress in the metabolism and functions of circRNAs and elaborately discuss the patterns of circRNA-protein interactions, including altering interactions between proteins, tethering or sequestering proteins, recruiting proteins to chromatin, forming circRNA-protein-mRNA ternary complexes and translocating or redistributing proteins. Many discoveries have revealed that circRNAs have unique expression signatures and play crucial roles in a variety of diseases, enabling them to potentially act as diagnostic biomarkers and therapeutic targets. This review systematically evaluates the roles and mechanisms of circRNAs, with the hope of advancing translational medicine involving circRNAs.
\end{abstract}

Keywords: CircRNA, CircRNA-protein interaction, Mechanism, Metabolism, Function

\section{Introduction}

Single-stranded, covalently closed circRNAs were first reported as viroids, which are pathogens of certain plants, in 1976 [1] and were first detected in human HeLa cells by electron microscopy in 1979 [2]. Later, more studies found or synthesized circular forms of RNAs in various species, including viruses [3], prokaryotes [4], unicellular eukaryotes $[4,5]$ and mammals [6]. With the development of high-throughput RNA-sequencing and bioinformatic tools, scientists have found that circRNA is a general feature of the human transcriptome and is ubiquitous in many other metazoans [7-9]. More recently, an increasing number of investigations have identified multiple functions of circRNAs, including

\footnotetext{
* Correspondence: juhq@sysucc.org.cn; xurh@sysucc.org.cn

${ }^{+}$Wei-Yi Zhou, Ze-Rong Cai and Jia Liu contributed equally to this work.

'State Key Laboratory of Oncology in South China, Collaborative Innovation

Center for Cancer Medicine, Sun Yat-sen University Cancer Center,

Guangzhou 510060, P. R. China

Full list of author information is available at the end of the article
}

serving as protein scaffolds or miR sponges and being translated into polypeptides $[7,8,10]$.

The unique structure of circRNAs provides them with a longer half-life and more resistance to RNase R than linear RNAs [11], which makes them potential candidates for diagnostic biomarkers and therapeutic targets. Plenty of studies have uncovered their distinct expression signatures and crucial biological roles in a variety of diseases, such as cancer [12-14], cardiovascular disease [15], neurological disorder [16] and autoimmune disease [17]. However, the mechanisms underlying the abnormal landscape of circRNAs and how circRNAs exert physiological or pathological roles in diseases remain poorly understood. Moreover, the majority of functional studies have shown that circRNAs act as miR sponges, which are monotonous and stereotypical. Antisense transcript of cerebellar degeneration-related protein 1 (CDR1as) represents the earliest functionally studied circRNAs and expands the competing endogenous RNA (ceRNA) 
crosstalk network [18-20]. Interestingly, a recent study reported that CDR1as interacts with IGF2BP3 and compromises its pro-metastatic functions [21]. Another one found that CDR1as interacts with p53 and blocks it from MDM2 [22]. This inspires us that circRNAs can be versatile and previously-studied circRNAs may also possess other abilities. Actually, one circRNA can simultaneously function as both a miR sponge and a protein template [23] (or interactor $[24,25])$.

Additionally, proteins are direct effectors of almost all vital activities. CircRNA-protein interactions have remarkably rejuvenated the field of circRNAs and enlightened our insights into their biological significance. However, studies on circRNA-protein interaction are still lacking, and its mechanisms fascinate scientists. Therefore, in this article, we reviewed the important progress in the metabolism and functions of circRNAs and highlighted the modes of circRNA-protein interactions. Hopefully, this review will help to reveal intricate circRNA-related issues and to develop circRNA-targeted translational research and clinical applications.

\section{Metabolism of circRNA}

In this module, we briefly introduce the cutting-edge investigations into circRNA metabolism and discuss how its modification influences metabolic programs (Fig. 1). To date, the upstream regulatory machinery of circRNA remains intriguing.

\section{Biogenesis}

Generally, pre-mRNA transcribed by RNA polymerase II (Pol II) contains introns and exons, followed by a 7methylguanosine cap and poly-adenosine tail adding to its $5^{\prime}$ - and $3^{\prime}$-ends, respectively. Then with the assistance of spliceosomes, pre-mRNA undergoes splicing at canonical splice sites (5'-GU and $3^{\prime}$-AG at introns) to become mature and translatable. CircRNA is generated by a special alternative splicing manner termed backsplicing, in which the $3^{\prime}$-end of an exon ligates to the $5^{\prime}$-end of its own or an upstream exon through a $3^{\prime}, 5^{\prime}$ phosphodiester bond, forming a closed structure with a back-splicing junction site $[7,8,26]$. Initially, circRNAs were regarded as splicing errors containing so-called "scrambled exons" [27].

According to the order of splicing events and different intermediates, two models of biogenesis were proposed [28] and validated [29]: the lariat model and the direct back-splicing model [26]. Recently, an excellent study assembled the spliceosome E complex on pre-mRNA and carried out structural and biochemical analyses, proposing an integral model for intron definition, exon definition, remodeling and the back-splicing-mediated circRNA biogenesis [30].

\section{Regulation}

CircRNA biogenesis relies on canonical splicing machinery, including splice signal sites and spliceosomes [31]. However, inhibiting the pre-mRNA processing machinery shifts the output of genes to circRNAs [32], which implies that there is competition between circRNAs and their linear counterparts. Cis-elements (intronic complementary sequences, ICSs) and trans-factors (RNA binding proteins, RBPs) can regulate circRNA production [33].

Inverted repeated Alu pairs in flanking introns facilitate exon circularization, while pairs in the same intron promote the extrusion of themselves and canonical splicing [34]. Mammalian-wide interspersed repeat family elements also contribute to the circularization of circRNAs [35]. Additionally, a group of RBPs modulate circRNA biogenesis by binding to flanking introns. Some directly draw introns into proximity and facilitate circularization [33, 36], and others stabilize [37] or impair [38] Alu pairs to promote or prevent back-splicing, respectively.

A recent study found that N6-methyladenosine (m6A) controls circRNA biogenesis. Methyltransferase-like 3 (METTL3) or YTH domain-containing 1 (YTHDC1) depletion both regulate approximately $20 \%$ of a subset of circRNAs, with no significant changes in the linear isoforms [39]. Another study found that alkB homolog 5 (ALKBH5) inhibition increases the production of translatable circRNAs through m6A enrichment at junction sites. Meanwhile, the m6A-modified start codon is recognized by YTHDF3 and mediates translation initiation [40]. However, whether there are any other regulatory factors and how m6A deposition affects the choice between back and canonical splicing remain unclear.

\section{Localization}

CircRNAs formed from exons are generally localized to the cytoplasm [41]. The mechanisms of their nuclear export remained elusive until a recent study found that UAP56 or URH49 depletion causes long or short circRNAs, respectively, to be enriched in the nucleus, which suggests that their transportation is partially length-dependent [42]. Another study showed that the nuclear export of circNSUN2 is mediated by YTHDC1 recruitment, which provides the first evidence that m6A controls circRNA translocation [43].

Additionally, scientists have identified certain introncontaining circRNAs that are retained in the nucleus and regulate their parental gene expression [44, 45]. Some exonic circRNAs are predominantly distributed in the nucleus as well and increase the nuclear retention of proteins [46] or recruit proteins to chromatin [47]. Moreover, circRNAs can be delivered by extracellular vesicle (EV) and detected in the circulation and urine [48]. The sorting of these exosomal circRNAs seems to 


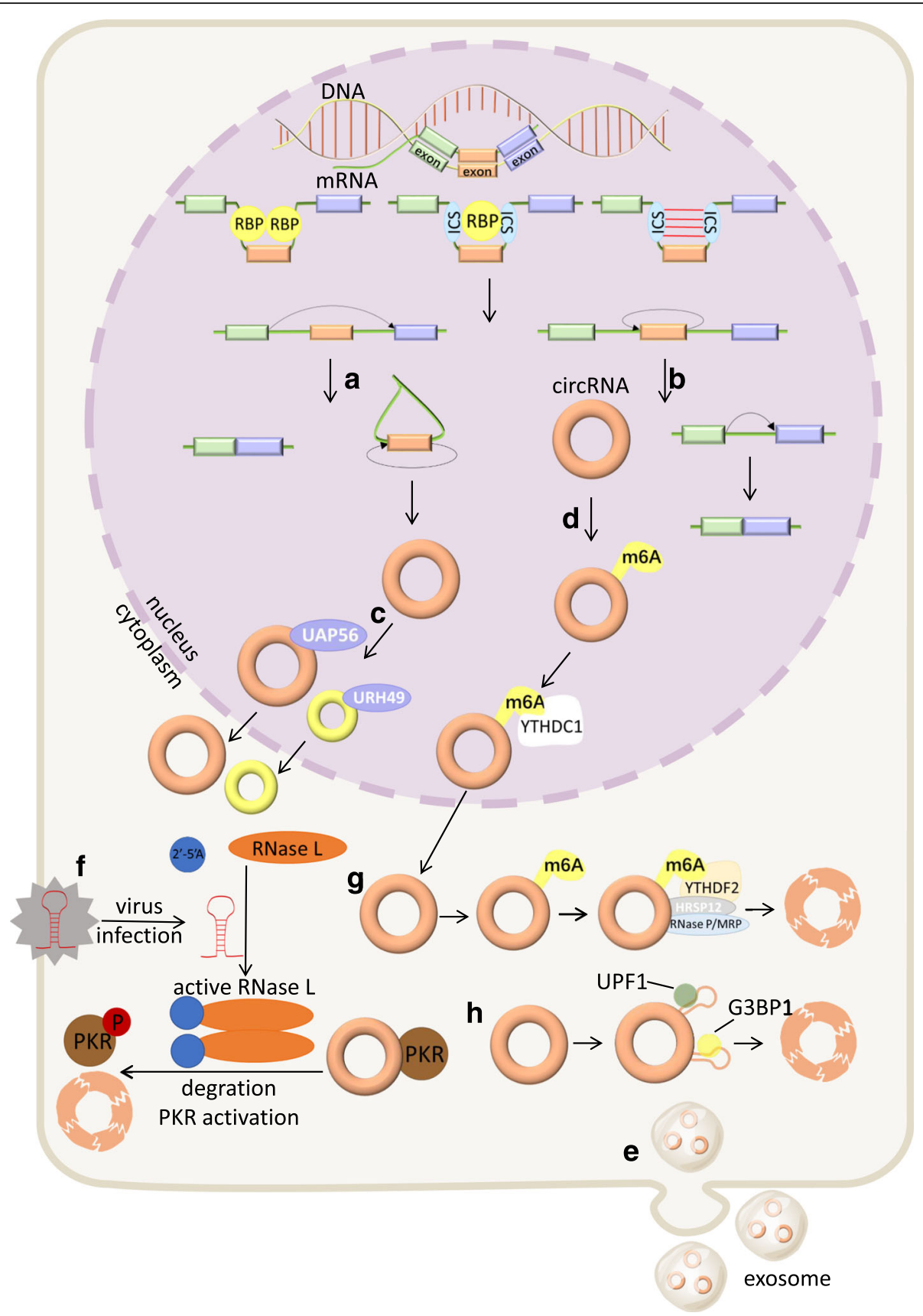

Fig. 1 Metabolism of circRNA. Regulation of circRNA biogenesis. RBP can modulate circRNA biogenesis by dimerization, ICS stabilization or ICS impairment. ICS in flanking introns can facilitate exon circularization. Biogenesis of circRNA. a In the lariat model, back-spliced exons are skipped and extruded to form an intronic lariat that undergoes further back-splicing, while the remaining exons directly link with each other and form a mature mRNA. $\mathbf{b}$ In the direct model, back-splicing occurs first to form a circRNA, leaving an immature linear RNA containing introns. Localization of circRNA cl Long (> $800 \mathrm{nt}$ ) or short circRNAs can be translocated to the cytoplasm with the assistance of UAP56 or URH49, respectively. $\mathbf{d}$ CircRNAs can be translocated to the cytoplasm in m6A-dependent manner mediated by YTHDC1. e CircRNAs can be excreted to the extracellular space by exosomes. Degradation of circRNA. $\mathbf{f}$ Upon viral infection, RNase $L$ activated by $2^{\prime}-5^{\prime}$-oligoadenosine $\left(2^{\prime}-5^{\prime} A\right)$ causes the global degradation of circRNAs, which relieves the suppression of PKR. $\mathbf{g}$ M6A-containing circRNAs can be recognized by YTHDF2, which interacts with the RNase P/ MRP complex bridged by HRSP12, and then the complex endoribonucleolytically cleaves circRNAs. $\mathbf{h}$ UPF1 and G3BP1 can bind to imperfect base-paired regions of circRNAs and induce their degradation 
be regulated by associated miR levels in producer cells, while the specific biological activities transferred to recipient cells are largely unknown in diverse settings [48, 49]. The packaging, delivery and absorption of them also remain elusive so far. Recently, a handful of studies identified mitochondria-located circRNAs and examined their functions, which broaden our knowledge of circRNA derivation and mitochondrial transcriptome [5052]. Nevertheless, circRNAs located in other organelles or subcellular compartments deserve further investigations.

\section{Degradation}

CircRNAs are stable and accumulate in many cell types, especially in neural tissues [53]. In contrast, another study reported a global reduction in circRNA abundance in highly proliferative tissues, possibly due to dilution by proliferation [54]. Regarding how circRNAs maintain a dynamic balance, recent research has shed light on the mechanisms of circRNA degradation. One study revealed that circRNAs can be globally degraded by RNase L. Endogenous circRNAs tend to form imperfect duplexes and inhibit PKR (dsRNA-activated protein kinase), while their reduction leads to aberrant PKR activation and autoimmunity [55]. Another study found that m6A-containing circRNAs are recognized by YTHD F2 that interacts with RNase P/MRP (mitochondrial RNA processing) bridged by heat-responsive protein 12 (HRSP12). Then, these circRNAs are endoribonucleolytically cleaved [56]. A more recent study proposed a structure-dependent mechanism mediated by UPF1 RNA helicase and ATPase (UPF1) and G3BP stress granule assembly factor 1 (G3BP1), which bind to highly structured base-paired regions and direct both mRNA and circRNA decay [57].

Additionally, miR-671 directs the cleavage of CDR1as in an Argonaute2 (Ago2)-dependent manner [58]. GW182 (a key component of P-body and RNAi machinery) is also involved in circRNA degradation [59]. Apart from intracellular pathways, circRNA excretion may contribute to their clearance [60]. Further studies are needed to fully understand circRNA decay mechanisms and interpret their homeostasis and differential distribution across cell types.

\section{Modification}

M6A is the most common RNA modification and is regulated by readers, writers and erasers [61]. Emerging studies have revealed a transcriptome-wide and celltype-specific map of m6A-containing circRNAs [62] and m6A involvement in circRNA metabolism [63]. In addition to abovementioned biogenesis [39, 40], nuclear export [43] and degradation [56], m6A also modulates circRNA translation. One study reported that m6A drives the translation initiation of circRNAs mediated by the interaction between eIF4G2 and YTHDF3, which is enhanced by METTL3/14 and suppressed by FTO [64]. Additionally, the YTHDF2 binding of m6A-markedselfcircRNAs abrogates innate circRNA immunity [65]. Other types of modifications in circRNAs await further identifications.

\section{Functions of circRNA}

In this module, we discuss how circRNAs function at the molecular level and underlying mechanisms mainly involve interactions with other molecules. CircRNAs have long been considered "non-coding" RNAs (ncRNAs) with regulatory potency $[20,66]$. Later, scientists identified translatable circRNAs [67, 68], and more recent studies presented evidence for their prevalence $[69,70]$ (Fig. 2). Briefly, downstream pathways of circRNA are mostly related to miR sponges and other brilliant talents that circRNA yields require brand new pursuits. We will systematically review the current progress in circRNA-protein interactions and discuss this topic in detail in the next module.

\section{Transcriptional regulation}

CircSEP3 originating from exon 6 of SEPALLATA3 increases the abundance of cognate exon 6-skipped variant by binding to the host DNA locus and forming an RNADNA hybrid or R-loop, resulting in transcriptional pausing and splicing factor recruitment [71]. Similarly, circSMARCA5 causes transcriptional termination at exon 15 of SMARCA5 through R-loop formation, upregulating the truncated nonfunctional isoform [72]. CircRNAs with introns retained between exons (exon-intron circRNAs, EIciRNAs) can combine with U1 small nuclear ribonucleoprotein through RNA-RNA interactions between snRNA and EIciRNAs and then interact with Pol II at parental gene promoters, enhancing their expression [44]. Likewise, circular intronic RNAs (ciRNAs) formed from lariats that escape debranching can accumulate at their synthesis sites and increase parental gene expression by modulating elongating Pol II activity [45].

\section{MicroRNA sponging}

Plenty of studies have confirmed that circRNAs exert biological functions by acting as ceRNAs or miR sponges [73, 74]. CDR1as contains 63 conserved miR-7 binding sites [20] and increases the levels of miR-7 target mRNAs, which are mostly associated with tumor progression $[75,76]$. By sponging miR-7, CDR1as regulates the development of zebrafish midbrain [20] and the processes of many other diseases [77]. Apart from miRs, circPan3 binds to and stabilizes the mRNA that encodes interleukin-13 (IL-13) receptor subunit IL-13R $\alpha 1$, eventually leading to the maintenance of intestinal stem cells 


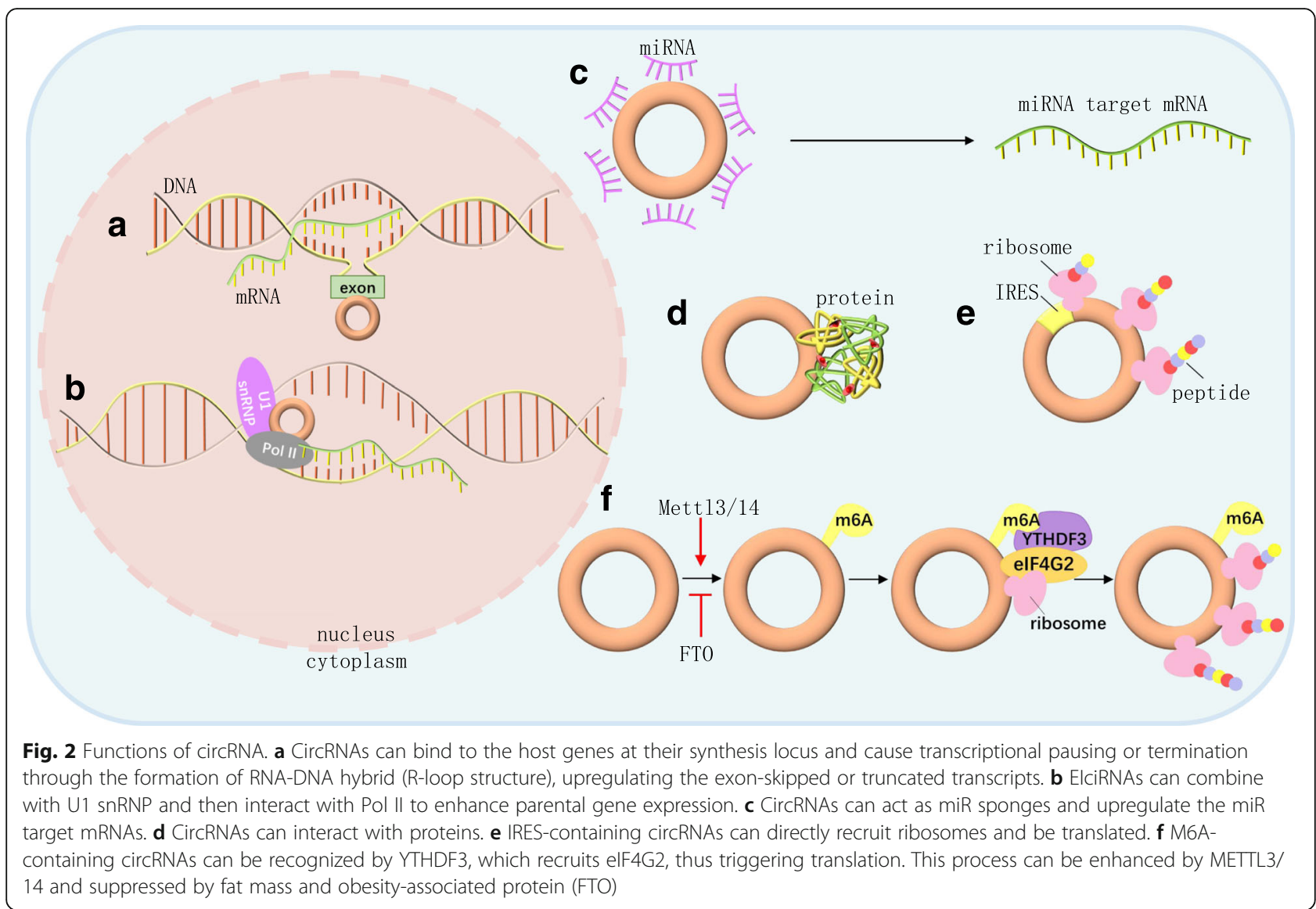

[78]. However, the stoichiometric relationship between circRNAs and other molecules (miRs and proteins) has received more attention $[7,13]$. In view of the generally low abundance of circRNAs and the limited number of binding sites, exactly how circRNAs exert sufficient/detectable effects remains unclear.

\section{Translation into proteins}

The translation is performed by ribosomes and involves initiation, elongation, termination and ribosome recycling [79]. The Initiation on eukaryotic mRNAs involves scanning by $43 \mathrm{~S}$ preinitiation complexes from the $5^{\prime}$ cap-proximal point of attachment to the initiation codon, followed by ribosomal subunit joining and factor displacement [80]. Lacking the 5'-cap and 3 '-tail, circRNA can only adopt cap-independent manners. In addition to previously described m6A-mediated translation [39, 64, 81], artificial [67] and endogenous circRNAs containing an internal ribosome entry site (IRES) that directly recruits ribosomes [82], can also be translated [63]. These two approaches may be coupled with each other. For example, m6A improves the efficiency of IRES-mediated translation of circZNF609 [39, 83]. Additionally, circRNA with an infinite ORF undergoes rolling circle amplification in an IRES-independent manner, leading to a hundred-fold higher productivity than linear transcript [84].

Peptides encoded by circRNAs are generally truncated and their functions are mostly analogous to the fulllength protein counterparts (circFBXW7-185aa [23, 85]). However, some proteins originating from circRNAs exert functions independent of or even opposed to those of their host gene products (circFNDC3B-218aa [86]). These results broaden the range of human proteome. However, the regulatory mechanisms of circRNA translation and the processes of elongation and termination are still not completely understood.

\section{CircRNA-protein interactions}

A handful of studies have reported that circRNAs function by interacting with proteins [87-89]. In addition to the abovementioned RBPs that participate in circRNA metabolism [33, 36], and circRNAs that interact with Pol II [44, 45], and miR-sponging circRNAs associated with Ago2 [58], a group of circRNAs serve as protein decoys, scaffolds and recruiters in diverse physiological and pathological contexts. One circRNA may exclusively bind to a single protein or interact with multiple proteins under different circumstances. One RBP can also combine with a subgroup of circRNAs and form 
circRNA-protein complex (circRNP) families (IGF2BP3) [90]. However, bioinformatic analyses indicated that circRNAs possess a lower RBP binding density than linear RNAs [91], which implies that many circRNAs are incapable of interacting with proteins. Thus, urgent solutions are needed to more efficiently carry out studies on circRNA-protein interactions. In this module, we classify circRNA-protein interactions into five sections (Fig. 3). Proteins involved in circRNA upstream processes (metabolism), and some special species (e.g., Ago2 and Pol II) will not be included (all relevant studies are listed in Table 1).

\section{Altering interactions between proteins}

In this section, we summarize three modes of circRNAprotein interactions: (1) one circRNA binds to both proteins and cements their interaction; (2) one circRNA binds to protein $\mathrm{A}$ and cements or dissociates its interaction with protein $\mathrm{B}$ that does not directly bind to circRNA; and (3) one circRNA binds to both proteins, which originally combine with each other, dissociating their interaction. The circRNA-protein A/B ternary (or more) complex arises in all three modes, but the effects are different.

\section{Cementing interactions between proteins}

In the first mode, circRNA mainly mediates posttranslational modifications (ubiquitination and phosphorylation) of protein $\mathrm{A}$ catalyzed by protein $\mathrm{B}$ or the transactivation of protein $\mathrm{A}$ by protein $\mathrm{B}$, followed by a subsequent downstream cascade. Silencing circFoxo3 reinforces cell viability, while its overexpression enhances tumor sensitivity to chemotherapy by potentiating apoptosis. Mechanistically, circFoxo3 interacts with p53 and mouse double minute 2 (MDM2), enhancing p53 ubiquitination. This also occupies MDM2 and relieves Foxo3 ubiquitination [92]. CircFoxo3 also increases Foxo3 translation by sponging miRs [161]. In addition, circFoxo3 is associated with cell cycle. The circFoxo3-p21CDK2 ternary complex reinforces the interaction of CDK2 with p21 (CDK inhibitor 1A) and dampens CDK2 phosphorylation activity. This impedes the formation of CDK2/cyclin E and CDK2/cyclin A complexes, and thus blocks G1/S transition and S progression, respectively, ultimately leading to cell cycle arrest in G1 phase [93]. This study addressed that a circRNA modulates proteinprotein interactions for the first time.

Similarly, the loss of circNfix promotes cardiomyocyte proliferation and angiogenesis, and inhibits apoptosis post myocardial infarction. Mechanistically, circNfix reinforces the interaction between Y-box binding protein 1 (YBX1) and NEDD4-like E3 ubiquitin ligase (Nedd4l), inducing YBX1 ubiquitination. CircNfix also inhibits the nuclear translocation of YBX1, which binds to the promoters of cyclin A2 and cyclin B1 and activates transcription. In addition, circNfix sponges miR-214 and increases glycogen synthase kinase 3 beta (GSK3 $\beta$ ) expression, subsequently repressing VEGF secretion and $\beta$-catenin activity [25]. Other examples of this mode, such as circADD3 [94], circAmotl1 [95], and circGLI1 [96] are listed in Table 1.

Regarding transactivation, circCTNNB1 promotes cancer progression by enhancing the transactivation of Yin Yang 1 (YY1) by DDX3 and thus upregulating target genes involved with $\beta$-catenin activation [97]. This mode is also illustrated by circCUX1, which promotes aerobic glycolysis and neuroblastoma progression by strengthening the transactivation of MYC-associated zinc finger protein (MAZ) by EWS RNA binding protein 1 (EWSR1) [98].

An outstanding study revealed that in metabolic adaptation to serum deprivation, the production of acetylCoA carboxylase 1 (ACC1) RNA switches from the linear to the circular form circACC1, which facilitates the assembly of the AMP-activated protein kinase (AMPK) holoenzyme by combining with the regulatory $\beta$ and $\gamma$ subunits and enhancing their interaction. This also stabilizes and activates AMPK, which inhibits anabolism and boosts catabolism, thus promoting $\beta$-oxidation and glycolysis. A tetramer is formed by AMPK and circACC1; however, circACC1 does not directly combine with the $\alpha$ subunit or enhance its interaction with another two subunits. The mechanism through which ACC1 pre-mRNA shifts to back-splicing under metabolic stress remains unexplored [99].

The second mode can be exemplified by circCcnb1. In p53 wild-type cells, circCcnb1 precipitates p53 bridged by $\mathrm{H} 2 \mathrm{~A}$.X variant histone ( $\mathrm{H} 2 \mathrm{AX})$, while in $\mathrm{p} 53$ mutant cells, it precipitates Bclaf1 also bridged by H2AX. However, circCcnb1 cannot directly access p53 or Bclaf1. The wild-type p53 has a greater affinity to H2AX than Bclaf1 and circCcnb1 augments this interaction, and thus the former ternary complex allows Bclaf1 to bind to $\mathrm{Bcl} 2$ and results in cell survival. While the mutant p53 is unable to bind to $\mathrm{H} 2 \mathrm{AX}$, and thus the latter complex wraps Bclaf1 by H2AX and results in cell death [100].

\section{Dissociating interactions between proteins}

In addition to $\mathrm{H} 2 \mathrm{AX}$, circCcnb1 can simultaneously interact with Ccnb1 and CDK1 and dissociates their interaction by forming a large ternary complex. This also decreases their nuclear translocation, thus arresting Ccnb1 function and decelerating cell cycle [101]. The situation in which a circRNA binds to protein A and dissociates its interaction with protein $B$ that does not directly bind to circRNA is sorted into the third type ("Blocking proteins from other proteins") of the next 
A

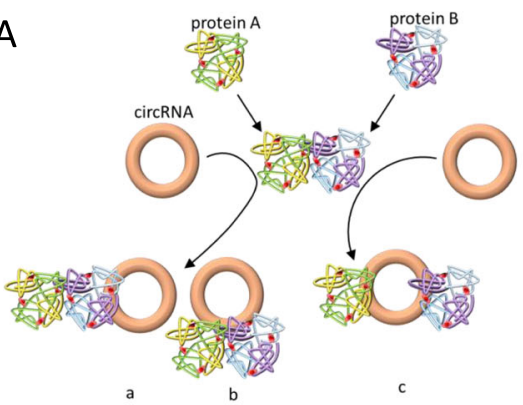

B

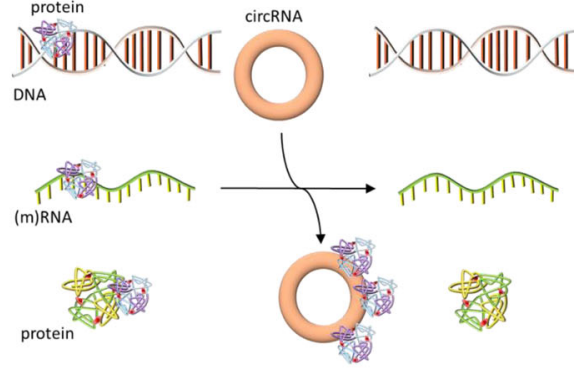

C
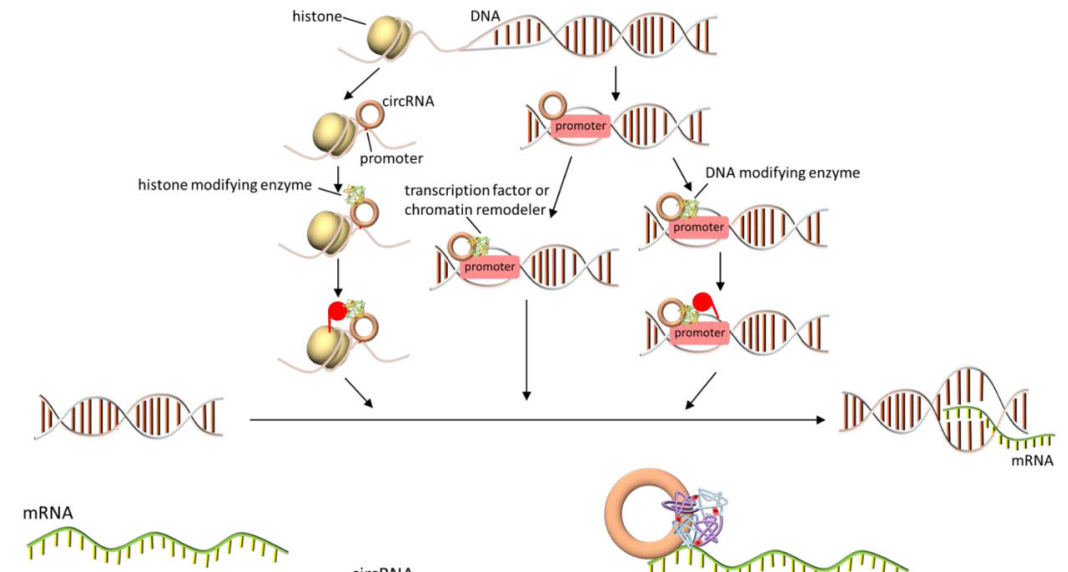

D
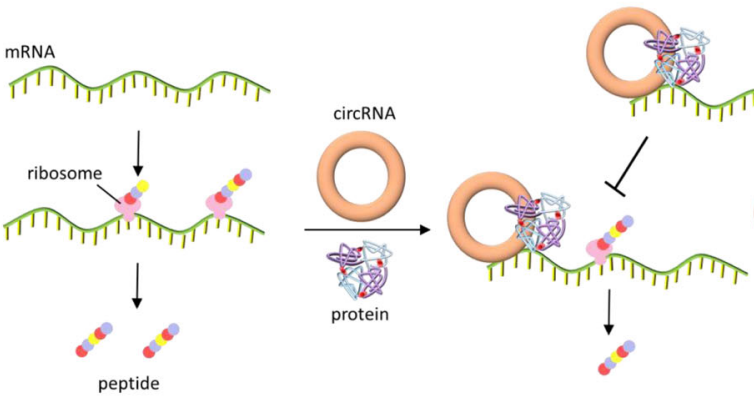

mRNA

peptide

transmembrane protein -

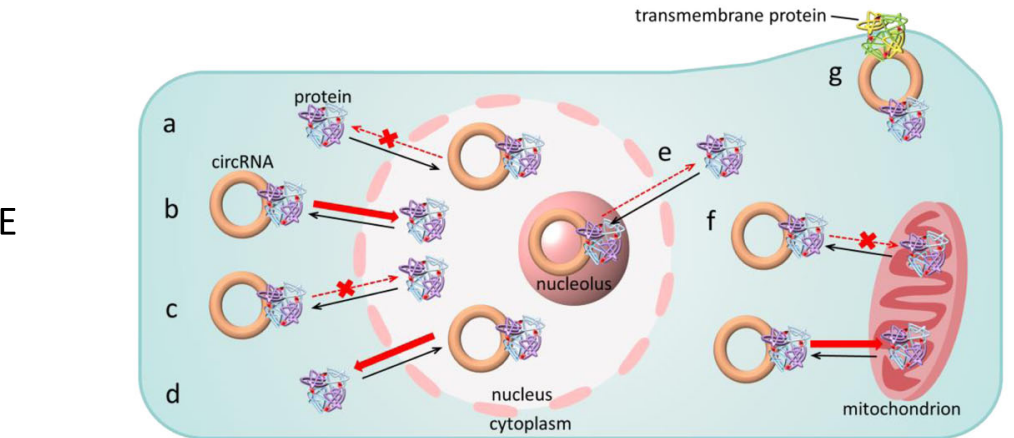

Fig. 3 CircRNA-protein interactions. A (a) CircRNA binds to both proteins and strengthens their interaction. (b) CircRNA binds to protein A and reinforces its interaction with protein B, which does not directly bind to circRNA. (c) CircRNA binds to both proteins that originally combine with each other and then disrupts their interaction. B CircRNA blocks proteins from interacting with DNA, RNA or other proteins, thus compromising their original functions. C CircRNA recruits transcription factors, chromatin remodelers and DNA or histone modifying enzymes to the promoters and alters transcription (including activating and inhibiting). D CircRNA helps RBPs to combine with mRNA and stabilizes mRNA (indirectly promoting translation) or directly regulates translation (including promoting and inhibiting). $\mathbf{E}$ (a) Nuclear circRNA causes the nuclear retention of proteins. (b) Cytoplasmic or shuttling circRNA facilitates the nuclear import of proteins. (c) Cytoplasmic circRNA causes the cytoplasmic retention of proteins. (d) Nuclear or shuttling circRNA facilitates the nuclear export of proteins. (e-g) Furthermore, circRNAs can transport proteins to the nucleolus, mitochondria and membrane, respectively 

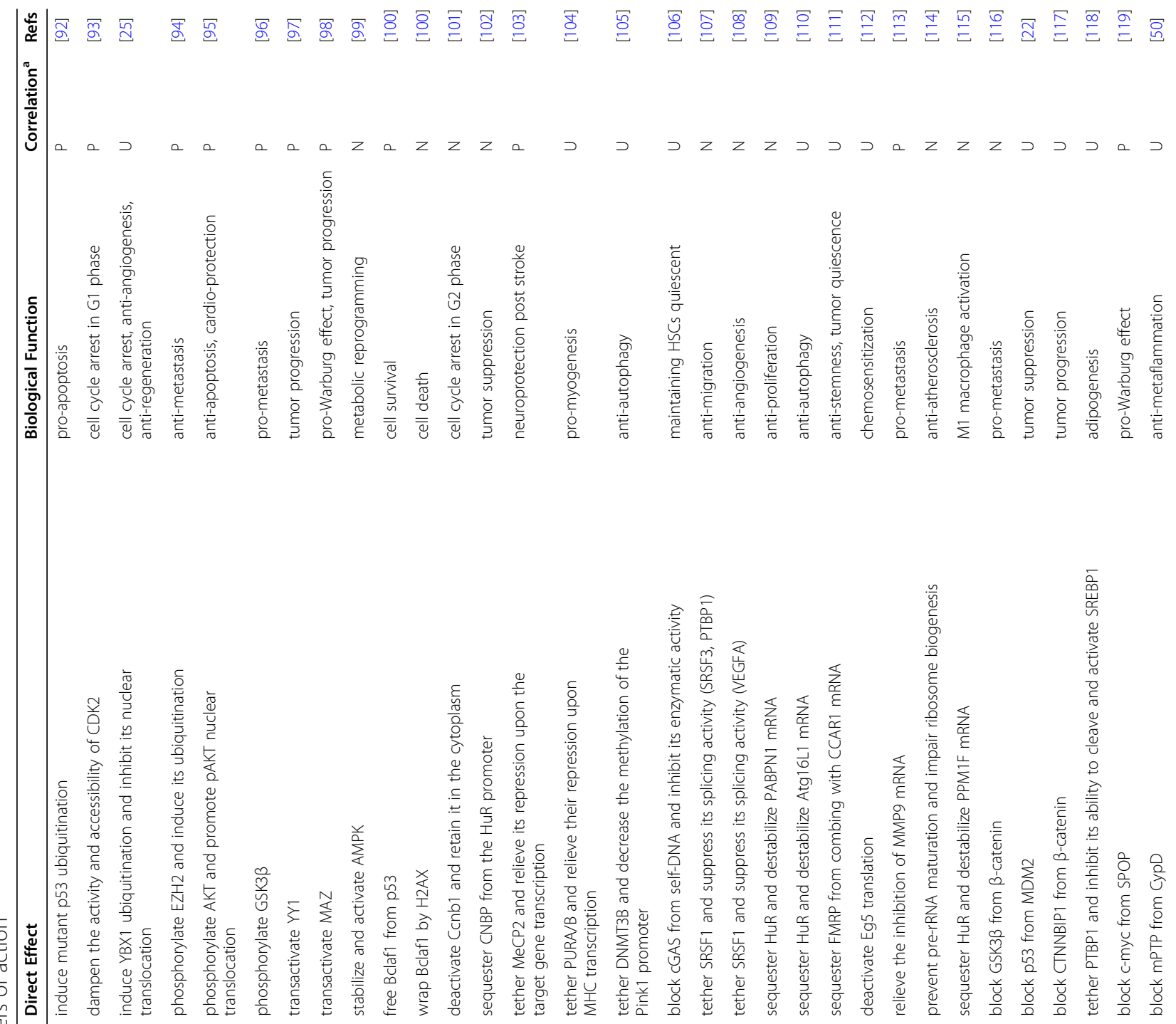

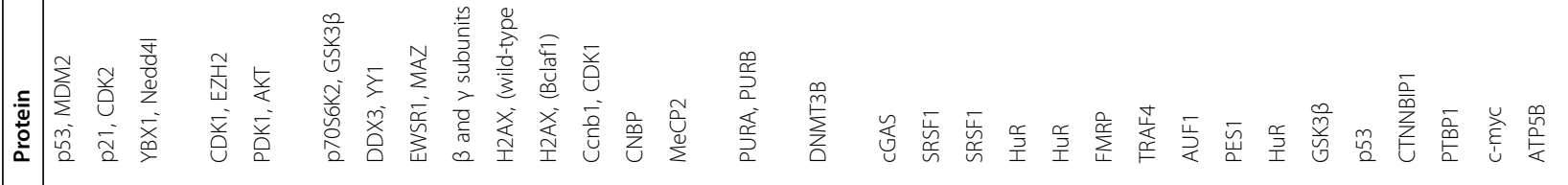

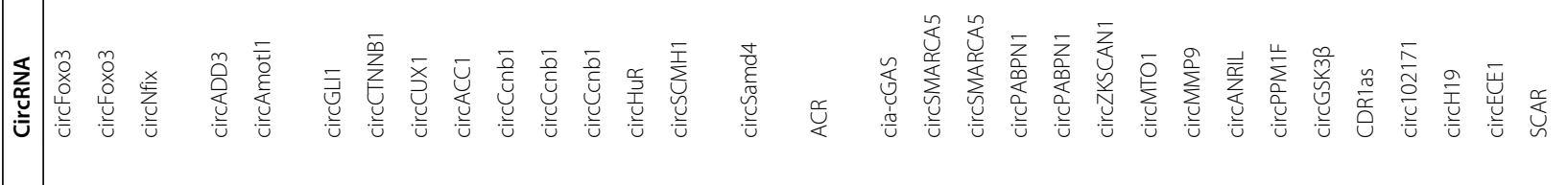



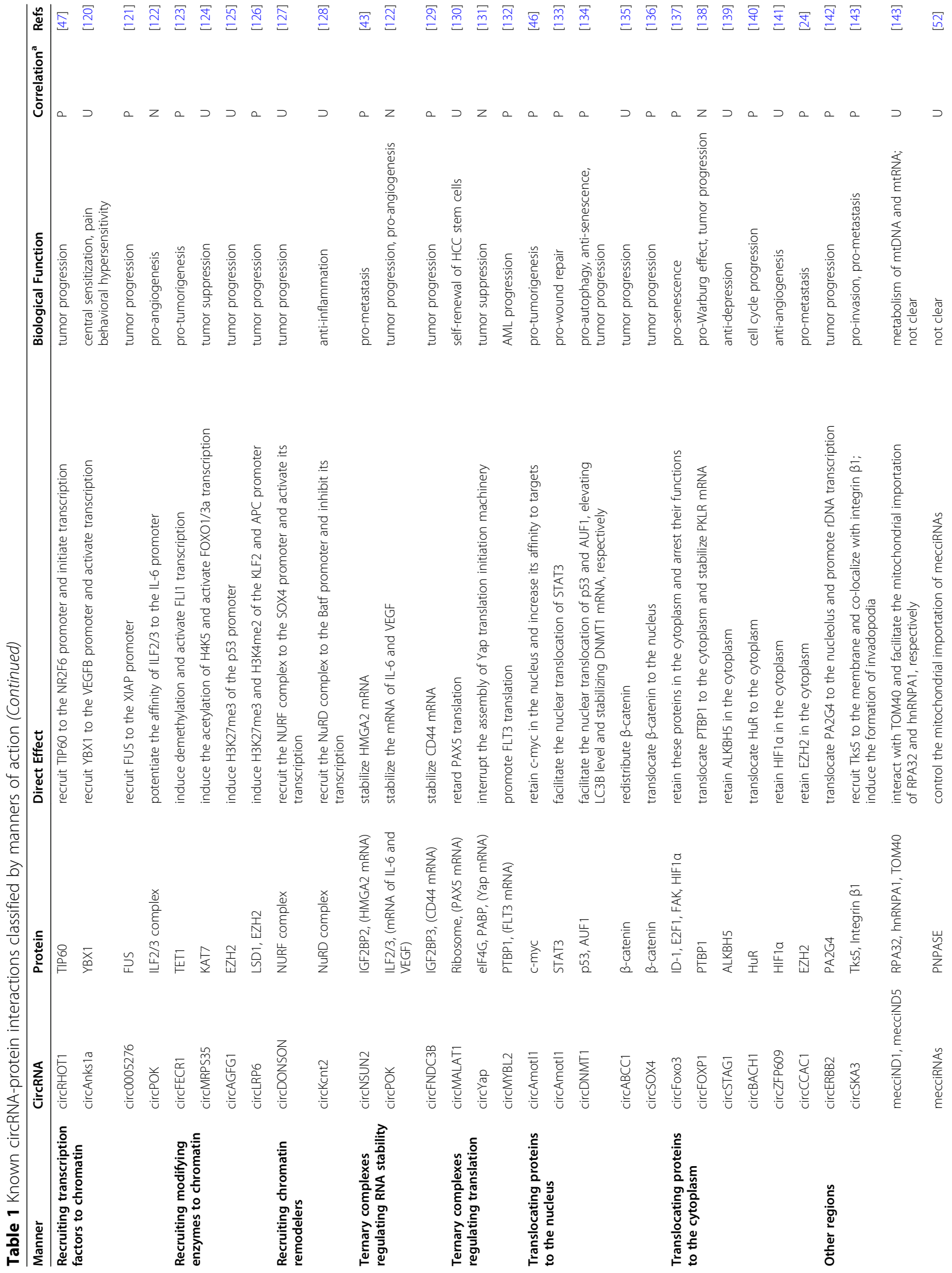

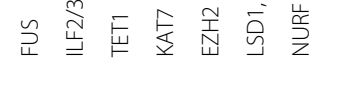




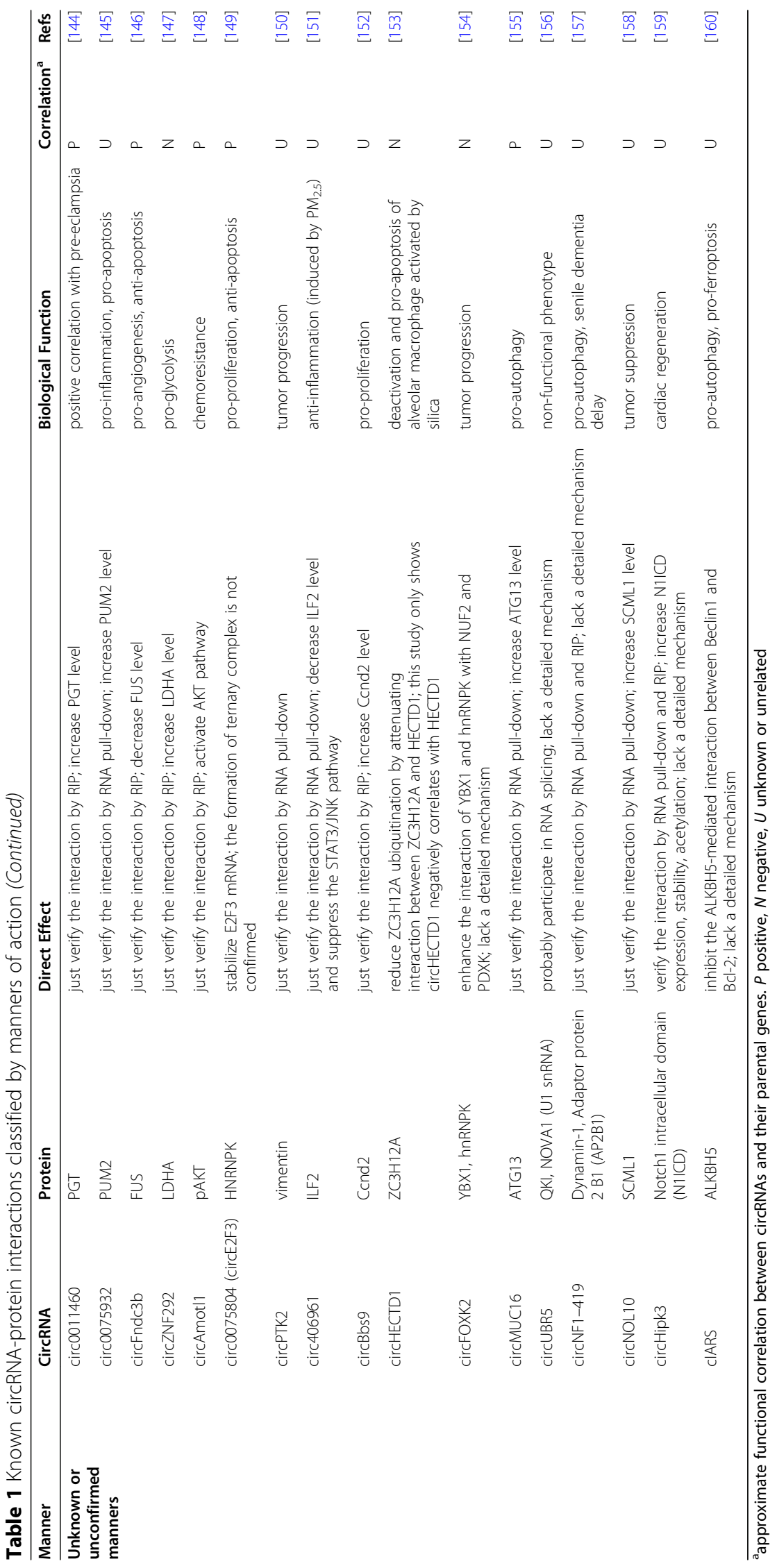


section. Actually, this disruption of interactions is the same as blocking protein A from B.

Therefore, we see the diversity of relationships between circRNA and two proteins and foresee the complications that arise when three or more proteins are involved. However, apart from mutual binding sites or sequences, little is known about exactly how circRNA alters protein-protein interactions. We speculate that circRNAs probably change the spatial distance of proteins, expose or cover their activity sites, and allosterically transform their conformation fit for interactions. More investigations and novel technologies are warranted to validate these hypotheses.

\section{Tethering or sequestering proteins}

In this section, circRNA combines with only one protein and compromises original function or creates new effects. We classify relevant studies into three types based on the downstream consequences: circRNA blocks proteins from interacting with DNA, RNA or other proteins.

\section{Blocking proteins from DNA}

In the first type, DNA binding proteins (e.g., transcription factors, TFs) represent the majority, and thus circRNA adversely alters transcription. CircHuR inhibits gastric cancer (GC) proliferation, invasion and metastasis by sequestering $\mathrm{CCHC}$-type zinc finger nucleic acid binding protein (CNBP) from the human antigen $R$ ( $\mathrm{HuR}$ ) promoter, thus downregulating $\mathrm{HuR}$ and repressing tumor [102]. Similarly, circSCMH1 delivered by EVs improves neuronal plasticity, and inhibits glial activation and immune cell infiltration post-stroke by sequestering methyl-CpG binding protein 2 (MeCP2) and compromising its role in transcriptional repression [103]. CircSamd4 [104] and autophagy-related circRNA (ACR) [105] also conform to this type.

Cyclic GMP-AMP (cGAMP) synthase (cGAS) is a DNA sensor that catalyzes cGAMP synthesis when it binds to DNA. Then, cGAMP activates the STING pathway and turn on type I interferon expression. This evolves as a defense mechanism against microbial infections, while cGAS activated by self-DNA triggers autoimmunity [162]. Under homeostatic conditions, circRNA antagonist for cGAS (cia-cGAS) occupies cGAS and avoids its binding with self-DNA. Meanwhile, this interaction inactivates synthase activity, thereby abrogating the cGAS-mediated production of type I interferon and protecting long-term hematopoietic stem cells (LTHSCs) from exhaustion [106].

\section{Blocking proteins from RNA}

The second type introduces a group of RBPs that control mRNA splicing, stability and translation. Therefore,
circRNA indirectly intervenes in posttranscriptional processes. CircSMARCA5 inhibits glioblastoma multiforme (GBM) cell migration by sequestering serine and arginine rich splicing factor 1 (SRSF1) that enhances exon 4 skipping in SRSF3 pre-mRNA. Both SRSF1 and SRSF3 (the isoform without exon 4) upregulate PTBP1, which contributes to glioma cell migration and adhesion [107, 163]. VEGFA pre-mRNA can also be alternatively spliced by SRSF1, and its aberrant splicing leads to an alteration in the ratio of the pro-/anti-angiogenic isoforms in GBM [108].

In addition to disrupting the splicing factor network, circPABPN1 sequesters $\mathrm{HuR}$ that stabilizes polyadenosine binding protein nuclear 1 (PABPN1) mRNA, consequently lowering PABPN1 levels and inhibiting HeLa cell proliferation [109]. It also prevents HuR from binding to autophagy-related 16 like $1 \mathrm{mRNA}$ and causes subsequent autophagy defects, triggering inflammatory bowel diseases [110]. Similar examples in which circRNAs indirectly regulate mRNA stability or translation include circZKSCAN1 [111], circMTO1 [112], circMMP9 [113] and circPPM1F [115].

CircANRIL appears to play an atheroprotective role against its linear counterpart linANRIL. The nucleolar protein pescadillo ribosomal biogenesis factor 1 (PES1) strongly interacts with circANRIL. By occupying the Cterminal lysine-rich domain of PES1, circANRIL prevents exonuclease-mediated pre-rRNA maturation and impairs ribosome biogenesis and assembly, leading to nucleolar stress. As a result, p53 is activated and accumulates in the nucleus, thus inducing apoptosis in atherosclerotic plaques [114].

\section{Blocking proteins from other proteins}

The third type has similarities to "Dissociating interactions between proteins" in the last section. In both of them, protein contact is broken. For example, circGSK3 $\beta$ promotes ESCC migration and invasion by decreasing $\beta$ catenin phosphorylation by GSK3 $\beta$ and its sequential ubiquitination [116]. CDR1as can block p53 from MDM2, thus relieving p53 ubiquitination and protecting cells from DNA damage [22]. Similar instances include circ102171 [117], circH19 [118] and circECE1 [119].

Recently, a breakthrough concerning mitochondrial genome-encoded circRNAs reported that SCAR (steatohepatitis-associated circRNA ATP5B regulator) can bind to ATP synthase subunit b (ATP5B) that resides in the mitochondrial permeability transition pore (MPTP) complex, and block mPTP from cyclophilin D, which shuts down MPTP and inhibits mitochondrial ROS output and fibroblast activation. However, endoplasmic reticulum stress induced by lipid overload (mimicking liver fibroblasts isolated from nonalcoholic steatohepatitis patients) represses PGC- $1 \alpha$ mediated by C/EBP 
homologous protein (CHOP), then downregulating SCAR [50].

However, the majority of current studies have merely observed the effects of circRNA sequestering proteins and have barely explored how circRNA influences proteins. Little is known about what happens to both molecules in this pattern (conformation, stability, abundance, distribution, modification).

\section{Recruiting proteins to chromatin}

In this section, several studies displayed novel patterns in which circRNAs bind to cis-elements and control TFs or modulate the epigenome, altering gene expression. We categorized them into three types: TFs, DNA or histone modifying enzymes and chromatin remodelers.

\section{Recruiting TFs}

Four examples showed that circRNAs recruit transcription activators to promoter regions. CircRHOT1 knockout suppresses hepatocellular carcinoma (HCC) proliferation, migration and invasion. It recruits Tat interactive protein $60 \mathrm{kDa}$ (TIP60) to the nuclear receptor subfamily 2 group F member 6 (NR2F6) promoter to initiate transcription [47]. Additionally, circAnks1a is upregulated in dorsal horn neurons following spinal nerve ligation and increases central sensitization and behavioral hypersensitivity. Mechanistically, cytoplasmic circAnks1a augments the transportin-1-mediated nuclear translocation of YBX1. Then, nuclear circAnks1a recruits YBX1 to the VEGFB promoter and activates transcription. Cytoplasmic circAnks1a also sponges miR-324-3p that targets VEGFB mRNA. Elevated VEGFB contributes to neuron excitability and pain behavior caused by nerve injury [120]. Circ0005276 [121] and circPOK [122] are also in this category.

\section{Recruiting modifying enzymes}

In regard to the epigenome, covalent modifying enzymes are recruited to DNA (methylation) or histones (methylation and acetylation) to alter chromatin accessibility and turn gene expression on or off. CircFECR1 enhances breast cancer (BC) invasion by recruiting ten-eleven translocation 1 (TET1) to the promoter of its parental gene Friend leukemia virus integration 1 (FLI1), inducing DNA demethylation and activating transcription [123]. Additionally, circMRPS35 suppresses GC proliferation and invasion by recruiting lysine acetyltransferase 7 (KAT7) to the FOXO1/3a promoter, which elicits H4K5 acetylation [124]. In contrast, circAGFG1 [125] and circLRP6 [126] both recruit enhancer of zeste 2 polycomb repressive complex 2 subunit (EZH2) to target gene promoters, inducing methylation and deactivating transcription.

\section{Recruiting chromatin remodelers}

CircDONSON promotes GC proliferation, migration and invasion by recruiting nucleosome remodeling factor (NURF, a chromatin remodeler complex) to the SRYbox transcription factor 4 (SOX4) promoter to activate its transcription [127]. In contrast, circKcnt2 inhibits the innate colitis by recruiting the nucleosome remodeling deacetylase (NuRD) complex to the Batf promoter to inhibit its transcription [128].

How cells acquire new identities or undergo reprogramming in response to various environmental cues has attracted attention. The interplay between TFs and threedimensional genome architecture triggers cell-fate decisions [164]. It will be of interest to determine how circRNAs shape the chromatin landscape and to examine the relationship between circRNAs and the transcriptome.

\section{Forming circRNA-protein-mRNA ternary complexes}

Ternary complexes are quite common in circRNAprotein interactions, and we mentioned circRNA-protein $\mathrm{A} / \mathrm{B}$ and circRNA-protein-chromatin complexes in the first and third sections, respectively. In this section, we focus on circRNA-protein-mRNA ternary complexes, which regulate mRNA stability or directly modulate translation. It is noteworthy that in circRNA-proteinnucleic acid (DNA or RNA) ternary complexes, three (or more) elements are combined together.

\section{Regulating stability}

The former case is conventional; circRNAs facilitate RBPs to combine with mRNAs, thus stabilizing mRNAs and increasing translation (no example of destabilizing so far). CircNSUN2 combines with IGF2BP2 and high mobility group A (HMGA2) mRNA, forming a ternary complex that stabilizes mRNA. Then, HMGA2 upregulation induces epithelial-mesenchymal transition and promotes colorectal cancer aggressiveness [43]. Additionally, circPOK functions antithetically to its linear counterpart that encodes Pokemon, which suppresses tumors. CircPOK interacts with interleukin enhancer binding factor $2 / 3$ (ILF2/3) complex and supports the stabilization of IL- 6 and VEGF mRNA by ILF2/3. It also potentiates the occupancy of ILF2/3 on the IL- 6 promoter. Collectively, circPOK regulates tumor cell secretome at both the transcriptional and posttranscriptional levels [122]. Similarly, the circFNDC3B-IGF2BP3-CD44 mRNA ternary complex stabilizes mRNA and upregulates CD44 [129].

\section{Regulating translation}

The latter case is more fascinating. One study described an unprecedented mode in which circRNA becomes stuck between mRNA and ribosome, acting like a brake and retarding translation. CircMALAT1 forms a ternary 
complex with paired box 5 (PAX5, a tumor suppressor) mRNA and ribosome through 11-complementary-bases and IRESs, respectively, leading to mRNA braking. It also sponges miR-6887-3p and activates the JAK2/ STAT3 signaling. Both pathways promote HCC stem cells self-renewal [130]. Another study introduced a circRNA that interposes the translation initiation machinery. CircYap combines not only with its linear counterpart Yap mRNA, but also with eIF4G and PABP, which attach to the $5^{\prime}$-cap and 3 '-tail, respectively. This tetramer obstructs the interaction between PABP and eIF4G, thus preventing Yap translation initiation [131]. Both studies depict how circRNA controls translation; however, one interrupts the elongation process, while another suppresses the assembly of initiation machinery. In contrast, circMYBL2 exacerbates acute myeloid leukemia by strengthening the interaction between PTBP1 and FMS-like tyrosine kinase 3 (FLT3) mRNA and promoting translation [132]. However, this study did not decipher how these molecules combine with each other (through binding domain, dynamic structure or sequence recognition?) and how this interaction improves translation efficiency.

Therefore, circRNAs exert biological roles at both the transcriptional/epigenetic and translational levels. Nevertheless, exact structures of the circRNA-protein-nucleic acid ternary complexes and precise processes of assembly, interconnection and possible disassembly have not been completely analyzed.

\section{Translocating or redistributing proteins}

The most common intracellular translocation events occur between the nucleus and cytoplasm. In this section, according to the circRNA localization and protein redistribution guided by circRNAs, we placed the situations into four categories: (1) nuclear circRNAs cause the nuclear retention of proteins; (2) cytoplasmic or shuttling circRNAs facilitate the nuclear import of proteins; (3) cytoplasmic circRNAs cause the cytoplasmic retention of proteins; and (4) nuclear or shuttling circRNAs facilitate the nuclear export of proteins. The former two lead to nuclear translocation, while the latter two result in the cytoplasmic translocation of proteins.

\section{Increasing nuclear distribution}

CircAmotll promotes BC proliferation and invasion, and inhibits apoptosis. Mechanistically, nuclear circAmotl1 directly binds to c-myc, increasing its nuclear retention and stability, and then enhances the affinity of c-myc to downstream gene promoters [46]. Another study showed that circAmotl1 interacts with STAT3 and facilitates its nuclear translocation. Nuclear STAT3 binds to the DNMT3a promoter and activates transcription. DNMT3a can methylate the miR-17 promoter, thus upregulating
miR-17-5p targets, including fibronectin, DNMT3a and STAT3. These factors form a positive feedback loop and promote fibroblasts proliferation, survival, adhesion, and migration, which together accelerate wound repair [133].

Similarly, circDNMT1 overexpression inhibits cellular senescence, which is mediated by stimulating autophagy. CircDNMT1 promotes the nuclear translocation of $\mathrm{p} 53$ and heterogeneous nuclear ribonucleoprotein D (hnRNPD, AUF1), inducing autophagy (by elevating LC3B level) and increasing DNMT1 translation (by stabilizing DNMT1 mRNA), respectively. Then, DNMT1 inhibits p53 transcription and decreases its total and cytoplasmic abundance while increasing its nuclear abundance [134]. Previous studies reported a dual role of p53 in controlling autophagy (cytoplasmic p53 represses autophagy, while nuclear p53 enhances autophagy) [165]. In turn, autophagy increases circDNMT1 levels, thus forming a complicated positive feedback network that suppresses senescence and promotes tumor progression [134]. Furthermore, circABCC1 [135] and circSOX4 [136] both increased the nuclear translocation of $\beta$-catenin and expedited tumor progression via the Wnt pathway.

\section{Increasing cytoplasmic distribution}

CircFoxo3 overexpression induces cardiac senescence and aggravates doxorubicin-induced cardiomyopathy by retaining the anti-senescence proteins inhibitor of DNA binding 1 (ID-1) and E2F1, as well as the anti-stress proteins tyrosine kinase 2 (FAK) and HIF1 $\alpha$ in the cytoplasm, disrupting the functions of these TFs, which mainly work in the nucleus (FAK in the mitochondria) [137]. Additionally, circFOXP1 promotes the Warburg effect and gallbladder progression by increasing the cytoplasmic translocation of PTBP1 that binds to the 3 'UTR and coding region of pyruvate kinase L/R (PKLR) mRNA, protecting it from decay [138]. Similarly, circSTAG1 ameliorated astrocyte dysfunction and depressivelike behaviors induced by chronic unpredictable stress by capturing ALKBH5 in the cytoplasm and increasing m6A levels of fatty acid amide hydrolase (FAAH) mRNA [139, 166]. CircBACH1 [140] and circZFP609 [141] also fall in this situation.

A recent study showed that circCCAC1 promotes cholangiocarcinoma (CCA) tumorigenesis by sponging miR-514a-5p that targets YY1. Additionally, it can be packed into EVs and transferred to endothelial monolayer cells, resulting in disrupted barrier integrity and enhanced angiogenesis. Mechanistically, circCCAC1 retains EZH2 in the cytoplasm, demethylating the $\mathrm{SH} 3$ domain containing the GRB2-like 2 (SH3GL2) promoter. Subsequently, intercellular junction proteins are decreased, and cell permeability is increased. However, this effect is absent in CCA cells. Therefore, circCCAC1 plays dual roles in CCA cells and 
endothelial cells by sponging miR and redistributing protein, respectively [24].

\section{Other intracellular regions}

Three studies illustrated that circRNAs transport proteins to the nucleolus, membrane and mitochondria, respectively. CircERBB2 promotes gallbladder cancer proliferation and mainly accumulates in the nucleolus. Ribosome synthesis is crucial for malignancy, and cancer is characterized by abnormal ribosomal DNA (rDNA) transcription by Pol I [167]. CircERBB2 increases the nucleolar localization of proliferation-associated 2G4 (PA2G4), promoting PA2G4-TIFIA (Pol I transcription factor) interaction and thereby recruiting Pol I to the rDNA promoter [168]. However, a more detailed mechanism of how circERBB2 moves PA2G4 and whether circERBB2 anchors other proteins involved in rDNA transcription remains unknown [142]. CircSKA3 enhances $\mathrm{BC}$ invasion and metastasis by recruiting Tks5 to the membrane. This induces the formation of invadopodia where circSKA3 co-localizes with actin, Tks5 and integrin $\beta 1$. Tks 5 does not directly bind to integrin $\beta 1$, but they precipitate each other, which indicates that a ternary complex bridged by circSKA3 forms [143].

A recent study found that mitochondria-encoded circRNAs (mecciRNAs) mecciND1 and mecciND5 can serve as molecular chaperones for replication protein A2 (RPA32) and hnRNPA1, respectively, and facilitate their entry into mitochondria by interacting with translocase of outer mitochondrial membrane 40 (TOM40). However, this effect in the posttranslational system is much weaker than that in the co-translational system, which suggests that mecciRNAs may only help newly synthesized peptides adopt structures that favor mitochondrial importation but have little impact on mature proteins [52]. MecciRNAs are distributed both inside and outside the mitochondria and may shuttle dynamically. Polynucleotide phosphorylase (PNPASE, a major mitochondrial RNA importation factor) interacts with most mecciRNAs and controls their mitochondrial levels [52].

In conclusion, protein redistribution is usually accompanied by enhanced or dampened activity and enabled or hampered access to targets, thus leading to boosted or arrested functions and corresponding downstream variations. However, the relationship between the transportation of proteins and circRNAs themselves has scarcely been studied.

\section{Other manners and summary}

Several other studies also verified circRNA-protein interactions. However, because detailed mechanistic research is lacking, we are unable to classify them into any manners (Table 1) [144-160]. Of note, the techniques of identifying and characterizing the dynamic circRNA- protein interactions have been systematically reviewed, which mainly include RBP immunoprecipitation (RIP), RNA pull-down, RNase protection assay (RPA), crosslinking immunoprecipitation (CLIP), electrophoretic mobility shift assay (EMSA), fluorescence in situ hybridization (FISH) and immunofluorescence. RIP followed by RNAsequencing/polymerase chain reaction profiles/verifies circRNAs that bind to specific proteins, while RNA pulldown followed by mass spectrometry/western blotting identifies/verifies proteins that bind to specific circRNAs. RPA and CLIP can map the sites of interactions, while EMSA can verify the formation of circRNA-protein complexes. FISH and immunofluorescence can detect the colocalization of circRNAs and proteins [8, 87-89].

Some circRNAs, such as circFOXO3 and circAmotl1, may implement diverse but similar functions by interacting with distinct proteins under different or dynamic circumstances. Some, such as circCcnb1 and circCCAC1, can even exert dual roles in different cell types. The underlying reason for this remains unknown and may be explained by external stimuli (specific microenvironmental or instructive factors) or internal features (dynamic tertiary structures or physicochemical properties). Some, such as circSMARCA5 and circPABPN1, may interact with the same protein that possesses various targets and thus be multifunctional.

It is necessary to state that five manners are not strictly exclusive, and occasional overlap appears among them. We distinguished them depending on the predominate effects observed and emphasized by researchers. Apart from the abovementioned ubiquity of ternary complexes, protein retention by circRNA is a sort of sequestration in some ways. Sometimes the formation of complexes occurs after the transportation of circRNPs, while sometimes alterations in protein activities or modifications lead to redistribution. Another aspect worth considering is the correlation between circRNA function and that of its parental gene. Nearly $50 \%$ circRNAs that interact with proteins act as positive regulators of parental genes, while approximately $20 \%$ act as negative regulators. The rest are unrelated or unknown yet. This phenomenon seems to not be subject to any specific rules. Some circRNAs directly interact with or indirectly regulate their cognate genes, linear counterpart transcripts or host gene products (DNA, mRNA and protein, respectively). However, most of them tread totally different pathways that lead to similar, opposite or unrelated outcomes compared to parental genes.

Interacting with the chief executors of life processes endows circRNAs with multiple biological functions. Among them, approximately $80 \%$ correlate with tumor progression or repression. Specifically, circRNAs engage in the overlapping modulation of tumorigenesis (proliferation and apoptosis), metastasis (invasion and migration), 
cell cycle, angiogenesis, metabolic reprogramming, senescence, autophagy, stemness, chemosensitivity, etc. The rest mainly include cardiovascular and neurological functions, inflammation and autoimmunity, wound repair and regeneration, myogenesis and adipogenesis, and undefined downstream effects. Though these studies involve extensive areas, they do not obey any other specific rules thus far, which indicates a large gap between basic research and clinical practice and reminds us that further explorations are indispensable.

\section{Conclusions and perspectives}

In this review, we briefly summarize recent progress in circRNA metabolism and functions. Among these, circRNA-protein interactions hold climbing appeal to scientists and still lack a comprehensive research framework. Therefore, we collected almost all relevant studies to date and classified them into five patterns according to the direct effects that circRNAs exert on proteins: (1) altering interactions between proteins; (2) tethering or sequestering proteins; (3) recruiting proteins to chromatin; (4) forming circRNA-protein-mRNA ternary complexes; and (5) translocating or redistributing proteins.

Despite fruitful progress, challenges and difficulties posed by theoretical deficiencies and technological restrictions exist. For example, what factors determine whether circRNAs act as scaffolds or decoys to support or impede proteins? Are there transcription repressors, other cis-elements (enhancers), chromatin modifiers or remodelers that are involved in circRNA regulation upon transcription? What upstream intrinsic properties or extrinsic factors elicit the formation of complexes from several specific molecules? Are there ncRNAs or other molecules (e.g., metabolites and ionic compounds) that combine with circRNPs? Are circRNAs involved in protein translocation to other organelles (e.g., the endoplasmic reticulum, Golgi apparatus and lysosome), subcellular compartments (e.g., euchromatic or heterochromatic territories, the nuclear lamina and membrane-less organelles formed by phase separation) or extracellular spaces (e.g., the matrix and adjacent or distant cells)? Do circRNAs affect the translocation of other molecular species? How can we profile circRNAs located in other organelles or deliver circRNAs to specific subcellular compartments? How can we analyze the exact structure of circRNPs beyond the binding sites to interpret the conformational and functional changes of proteins? How can we improve the canonical techniques (e.g. RIP, RNA pull-down, RNAsequencing) in view of the low abundance and special structure of circRNAs? How can we capture the realtimecircRNA-protein interactions in vivo and display the dynamic processes? How can we design the animal models and conduct pre-clinical studies targeting circRNPs?
At present, the study on circRNA-protein interaction is still staying at the very beginning stage and far away from translational medicine. Though their unique structures and resistance to RNA decay machinery enable them to act as ideal diagnostic biomarkers and therapeutic targets, regrettably, not a single circRNA-based medical application has been approved so far. However, some excellent studies that we have mentioned above have shown promising results and achievements. For example, in the study of circRNA SCAR, a mitochondria-targeting nanoparticle (mito-NP) platform that specifically delivers encapsulated vectors to the mitochondria was designed and this enables mitochondria-targeted therapy to tackle metaflammation associated diseases [50]. In the study of circPan3, cia-cGAS and circKcnt2, the researchers generated circRNA-knockout mice by targeting the ICSs in flanking introns of circRNAs with CRISPR/ Cas9 technology, which avoids affecting corresponding parental genes [78, 106, 128]. This provides convenience for basic research and possibilities for translational practice. CircCcnb1 has totally inverse roles in different cells, which makes it a potential therapeutic agent for p53 mutant tumors because it causes less damage to adjacent benign p53 wild-type tissues [100]. Proteins control the biological activities in all aspects. CircRNA-protein interactions make changes to proteins and thus make a difference in life processes, which sparks our interest in devising novel clinical strategies that manipulate (interfere or utilize) circRNAs based on circRNA-protein interaction.

\section{Abbreviations}

circRNA: circular RNA; miR: microRNA; mRNA: messenger RNA;

CDR1as: Antisense transcript of cerebellar degeneration-related protein 1; ceRNA: competing endogenous RNA; Pol II: RNA polymerase II; ICS: intronic complementary sequence; RBP: RNA binding protein; m6A: N6methyladenosine; METTL: Methyltransferase-like; YTHDC: YTH domaincontaining; YTHDF: YTH domain family; EV: extracellular vesicle; HCC: hepatocellular carcinoma; Ago2: Argonaute2; elF4G: eukaryotic translation initiation factor 4 gamma; ncRNA: non-coding RNA; IncRNA: long non-coding RNA; IGF2BP: insulin like growth factor 2 mRNA binding protein; IL: interleukin; IRES: internal ribosome entry site; UTR: untranslated region; ORF: open reading frame; circRNP: circRNA-protein complex; MDM2: mouse double minute 2; FOXO3: forkhead box O3; CDK: cyclin-dependent kinase;

YBX1: Y-box binding protein 1; Ccn: cyclin; GSK33: glycogen synthase kinase 3 beta; VEGF: vascular endothelial growth factor; ACC1: acetyl-CoA

carboxylase 1; AMPK: AMP-activated protein kinase; TF: transcription factor; GC: gastric cancer; HuR: human antigen R; CGAS: cyclic GMP-AMP synthase; SRSF: serine and arginine rich splicing factor; PTBP: polypyrimidine tract binding protein; PABP: poly-adenosine binding protein; BC: breast cancer; DNMT: DNA methyltransferase; ILF: interleukin enhancer binding factor; Yap: Yes1-associated transcriptional regulator; STAT3: signal transducer and activator of transcription 3; LC3B: light chain 3 beta; Pol I: RNA polymerase I; mecciRNAs: mitochondria-encoded circRNAs; hnRNP: heterogeneous nuclear ribonucleoprotein

\section{Acknowledgements}

Not applicable. 


\section{Authors' contributions}

Conceptualization: Xu RH and Ju HQ; Writing-Review \& Editing: Zhou WY, CAI ZR, Liu J, Wang DS and Ju HQ; Supervision: Xu RH and Ju HQ. All authors read and approved the final manuscript.

\section{Funding}

This research was supported by the National Key R\&D Program of China (, 2018YFC1313300, 2018YFC1313304), National Natural Science Foundation of China (81930065, 81871951), Natural Science Foundation of Guangdong Province (2019A1515010233, 2018B030306049), Pearl River S\&T Nova Program of Guangzhou (201806010002) and CAMS Innovation Fund for Medical Sciences (CIFMS) (2019-I2M-5-036).

\section{Availability of data and materials}

Not applicable.

Ethics approval and consent to participate

Not applicable.

\section{Consent for publication}

Not applicable.

\section{Competing interests}

The authors declare that they have no competing interests.

\section{Author details}

${ }^{1}$ State Key Laboratory of Oncology in South China, Collaborative Innovation Center for Cancer Medicine, Sun Yat-sen University Cancer Center, Guangzhou 510060, P. R. China. ${ }^{2}$ Research Unit of Precision Diagnosis and Treatment for Gastrointestinal Cancer, Chinese Academy of Medical Sciences, Guangzhou 510060, P. R. China.

\section{Received: 6 September 2020 Accepted: 20 November 2020} Published online: 14 December 2020

\section{References}

1. Sanger HL, Klotz G, Riesner D, Gross HJ, Kleinschmidt AK. Viroids are singlestranded covalently closed circular RNA molecules existing as highly basepaired rod-like structures. Proc Natl Acad Sci U S A. 1976;73(11):3852-6.

2. Hsu MT, Coca-Prados M. Electron microscopic evidence for the circular form of RNA in the cytoplasm of eukaryotic cells. Nature. 1979;280(5720):339-40.

3. Kos A, Dijkema R, Arnberg AC, van der Meide PH, Schellekens H. The hepatitis delta (delta) virus possesses a circular RNA. Nature. 1986;323(6088): $558-60$

4. Ford E, Ares MJ. Synthesis of circular RNA in bacteria and yeast using RNA cyclase ribozymes derived from a group I intron of phage T4. Proc Natl Acad Sci U S A. 1994;91(8):3117-21.

5. Grabowski PJ, Zaug AJ, Cech TR. The intervening sequence of the ribosomal RNA precursor is converted to a circular RNA in isolated nuclei of Tetrahymena. Cell. 1981;23(2):467-76.

6. Capel B, Swain A, Nicolis S, Hacker A, Walter M, Koopman P, et al. Circular transcripts of the testis-determining gene Sry in adult mouse testis. Cell. 1993;73(5):1019-30.

7. Chen LL. The expanding regulatory mechanisms and cellular functions of circular RNAs. Nat Rev Mol Cell Biol. 2020;21(8):475-90.

8. Kristensen LS, Andersen MS, Stagsted L, Ebbesen KK, Hansen TB, Kjems J. The biogenesis, biology and characterization of circular RNAs. Nat Rev Genet. 2019;20(11):675-91.

9. Szabo L, Salzman J. Detecting circular RNAs: bioinformatic and experimental challenges. Nat Rev Genet. 2016;17(11):679-92.

10. Xiao MS, Ai Y, Wilusz JE. Biogenesis and functions of circular RNAs come into focus. Trends Cell Biol. 2020;30(3):226-40.

11. Jeck WR, Sharpless NE. Detecting and characterizing circular RNAs. Nat Biotechnol. 2014;32(5):453-61.

12. Vo JN, Cieslik M, Zhang Y, Shukla S, Xiao L, Zhang Y, et al. The landscape of circular RNA in Cancer. CELL. 2019;176(4):869-81.

13. Li J, Sun D, Pu W, Wang J, Peng Y. Circular RNAs in Cancer: biogenesis, function, and clinical significance. Trends Cancer. 2020;6(4):319-36.

14. Ju HQ, Zhao Q, Wang F, Lan P, Wang Z, Zuo ZX, et al. A circRNA signature predicts postoperative recurrence in stage II/III colon cancer. EMBO Mol Med. 2019;11(10):e10168.
15. Aufiero S, Reckman YJ, Pinto YM, Creemers EE. Circular RNAs open a new chapter in cardiovascular biology. Nat Rev Cardiol. 2019;16(8):503-14.

16. Mehta SL, Dempsey RJ, Vemuganti R. Role of circular RNAs in brain development and CNS diseases. Prog Neurobiol. 2020;186:101746.

17. Zhou Z, Sun B, Huang S, Zhao L. Roles of circular RNAs in immune regulation and autoimmune diseases. Cell Death Dis. 2019;10(7):503.

18. Tay Y, Rinn J, Pandolfi PP. The multilayered complexity of ceRNA crosstalk and competition. Nature. 2014;505(7483):344-52.

19. Bosson AD, Zamudio JR, Sharp PA. Endogenous miRNA and target concentrations determine susceptibility to potential ceRNA competition. Mol Cell. 2014;56(3):347-59.

20. Memczak S, Jens M, Elefsinioti A, Torti F, Krueger J, Rybak A, et al. Circular RNAs are a large class of animal RNAs with regulatory potency. Nature. 2013;495(7441):333-8.

21. Hanniford D, Ulloa-Morales A, Karz A, Berzoti-Coelho MG, Moubarak RS, Sanchez-Sendra B, et al. Epigenetic silencing of CDR1as drives IGF2BP3mediated melanoma invasion and metastasis. Cancer Cell. 2020;37(1):55-70.

22. Lou J, Hao Y, Lin K, Lyu Y, Chen M, Wang H, et al. Circular RNA CDR1 as disrupts the p53/MDM2 complex to inhibit Gliomagenesis. Mol Cancer. 2020;19(1):138.

23. Ye F, Gao G, Zou Y, Zheng S, Zhang L, Ou X, et al. circFBXW7 inhibits malignant progression by sponging miR-197-3p and encoding a 185-aa protein in triple-negative breast Cancer. Mol Ther Nucleic Acids. 2019;18:88-98.

24. Xu Y, Leng K, Yao Y, Kang P, Liao G, Han Y, et al. A novel circular RNA, circCCAC1, contributes to CCA progression, induces angiogenesis, and disrupts vascular endothelial barriers. Hepatology. 2020.

25. Huang S, Li X, Zheng H, Si X, Li B, Wei G, et al. Loss of super-enhancerregulated circRNA Nfix induces cardiac regeneration after myocardial infarction in adult mice. Circulation. 2019;139(25):2857-76.

26. Chen LL, Yang L. Regulation of circRNA biogenesis. RNA Biol. 2015;12(4): 381-8.

27. Nigro JM, Cho KR, Fearon ER, Kern SE, Ruppert JM, Oliner JD, et al. Scrambled exons. Cell. 1991;64(3):607-13.

28. Zaphiropoulos PG. Circular RNAs from transcripts of the rat cytochrome P450 2C24 gene: correlation with exon skipping. Proc Natl Acad Sci U S A. 1996;93(13):6536-41.

29. Jeck WR, Sorrentino JA, Wang K, Slevin MK, Burd CE, Liu J, et al. Circular RNAs are abundant, conserved, and associated with ALU repeats. RNA. 2013; 19(2):141-57.

30. Li X, Liu S, Zhang L, Issaian A, Hill RC, Espinosa S, et al. A unified mechanism for intron and exon definition and back-splicing. Nature. 2019;573(7774):375-80.

31. Starke $S$, Jost I, Rossbach O, Schneider T, Schreiner S, Hung LH, et al. Exon circularization requires canonical splice signals. Cell Rep. 2015;10(1):103-11.

32. Liang D, Tatomer DC, Luo Z, Wu H, Yang L, Chen LL, et al. The output of protein-coding genes shifts to circular RNAs when the pre-mRNA processing machinery is limiting. Mol Cell. 2017;68(5):940-54.

33. Ashwal-Fluss R, Meyer M, Pamudurti NR, Ivanov A, Bartok O, Hanan M, et al. circRNA biogenesis competes with pre-mRNA splicing. Mol Cell. 2014;56(1): $55-66$.

34. Zhang XO, Wang HB, Zhang Y, Lu X, Chen LL, Yang L. Complementary sequence-mediated exon circularization. Cell. 2014;159(1):134-47.

35. Yoshimoto R, Rahimi K, Hansen TB, Kjems J, Mayeda A. Biosynthesis of Circular RNA ciRS-7/CDR1as is Mediated by Mammalian-wide Interspersed Repeats. iscience. 2020;23(7):101345.

36. Conn SJ, Pillman KA, Toubia J, Conn VM, Salmanidis M, Phillips CA, et al. The RNA binding protein quaking regulates formation of circRNAs. Cell. 2015; 160(6):1125-34.

37. Li X, Liu CX, Xue W, Zhang Y, Jiang S, Yin QF, et al. Coordinated circRNA biogenesis and function with NF90/NF110 in viral infection. Mol Cell. 2017; 67(2):214-27.

38. Aktas T, Avsar II, Maticzka D, Bhardwaj V, Pessoa RC, Mittler G, et al. DHX9 suppresses RNA processing defects originating from the Alu invasion of the human genome. Nature. 2017;544(7648):115-9.

39. Di Timoteo G, Dattilo D, Centron-Broco A, Colantoni A, Guarnacci M, Rossi F, et al. Modulation of circRNA metabolism by $\mathrm{m}^{6} \mathrm{~A}$ modification. Cell Rep. 2020;31(6):107641.

40. Tang C, Xie Y, Yu T, Liu N, Wang Z, Woolsey RJ, et al. $\mathrm{m}^{6} \mathrm{~A}$-dependent biogenesis of circular RNAs in male germ cells. Cell Res. 2020;30(3):211-28

41. Salzman J, Gawad C, Wang PL, Lacayo N, Brown PO. Circular RNAs are the predominant transcript isoform from hundreds of human genes in diverse cell types. PLoS One. 2012;7(2):e30733. 
42. Huang C, Liang D, Tatomer DC, Wilusz JE. A length-dependent evolutionarily conserved pathway controls nuclear export of circular RNAs. Genes Dev. 2018;32(9-10):639-44.

43. Chen RX, Chen X, Xia LP, Zhang JX, Pan ZZ, Ma XD, et al. $N^{6}$ methyladenosine modification of circNSUN2 facilitates cytoplasmic export and stabilizes HMGA2 to promote colorectal liver metastasis. Nat Commun. 2019;10(1):4695

44. Li Z, Huang C, Bao C, Chen L, Lin M, Wang X, et al. Exon-intron circular RNAs regulate transcription in the nucleus. Nat Struct Mol Biol. 2015;22(3): 256-64.

45. Zhang $Y$, Zhang $X O$, Chen $T$, Xiang JF, Yin QF, Xing YH, et al. Circular intronic long noncoding RNAs. Mol Cell. 2013;51(6):792-806.

46. Yang Q, Du WW, Wu N, Yang W, Awan FM, Fang L, et al. A circular RNA promotes tumorigenesis by inducing c-myc nuclear translocation. Cell Death Differ. 2017:24(9):1609-20.

47. Wang L, Long H, Zheng Q, Bo X, Xiao X, Li B. Circular RNA circRHOT1 promotes hepatocellular carcinoma progression by initiation of NR2F6 expression. Mol Cancer. 2019;18(1):119.

48. Wang Y, Liu J, Ma J, Sun T, Zhou Q, Wang W, et al. Exosomal circRNAs: biogenesis, effect and application in human diseases. Mol Cancer. 2019; 18(1):116.

49. Li Y, Zheng Q, Bao C, Li S, Guo W, Zhao J, et al. Circular RNA is enriched and stable in exosomes: a promising biomarker for cancer diagnosis. Cell Res. 2015;25(8):981-4.

50. Zhao Q, Liu J, Deng H, Ma R, Liao JY, Liang H, et al. Targeting mitochondrialocated circRNA SCAR alleviates NASH via reducing mROS output. Cell. 2020;183(1):76-93.

51. Wu Z, Sun H, Wang C, Liu W, Liu M, Zhu Y, et al. Mitochondrial genomederived circRNA mc-COX2 functions as an Oncogene in chronic lymphocytic leukemia. Mol Ther Nucleic Acids. 2020;20:801-11.

52. Liu $X$, Wang $X, L i J$, Hu S, Deng $Y$, Yin $H$, et al. Identification of mecciRNAs and their roles in the mitochondrial entry of proteins. Sci China Life Sci. 2020;63(10):1429-49.

53. Rybak-Wolf A, Stottmeister C, Glazar P, Jens M, Pino N, Giusti S, et al. Circular RNAs in the mammalian brain are highly abundant, conserved, and dynamically expressed. Mol Cell. 2015;58(5):870-85.

54. Bachmayr-Heyda A, Reiner AT, Auer K, Sukhbaatar N, Aust S, BachleitnerHofmann T, et al. Correlation of circular RNA abundance with proliferation-exemplified with colorectal and ovarian cancer, idiopathic lung fibrosis, and normal human tissues. Sci Rep. 2015;5:8057.

55. Liu CX, Li X, Nan F, Jiang S, Gao X, Guo SK, et al. Structure and degradation of circular RNAs regulate PKR activation in innate immunity. Cell. 2019; 177(4):865-80.

56. Park OH, Ha H, Lee $\mathrm{Y}$, Boo SH, Kwon DH, Song HK, et al. Endoribonucleolytic cleavage of $\mathrm{m}^{6} \mathrm{~A}$-containing RNAs by RNase P/MRP complex. Mol Cell. 2019; 74(3):494-507

57. Fischer JW, Busa VF, Shao Y, Leung A. Structure-mediated RNA decay by UPF1 and G3BP1. Mol Cell. 2020;78(1):70-84

58. Hansen TB, Wiklund ED, Bramsen JB, Villadsen SB, Statham AL, Clark SJ, et al. miRNA-dependent gene silencing involving Ago2-mediated cleavage of a circular antisense RNA. EMBO J. 2011;30(21):4414-22.

59. Jia R, Xiao MS, Li Z, Shan G, Huang C. Defining an evolutionarily conserved role of GW182 in circular RNA degradation. Cell Discov. 2019:5:45.

60. Lasda E, Parker R. Circular RNAs co-precipitate with extracellular vesicles: a possible mechanism for circRNA clearance. PLoS One. 2016;11(2):e148407.

61. Zaccara S, Ries RJ, Jaffrey SR. Reading, writing and erasing mRNA methylation. Nat Rev Mol Cell Biol. 2019;20(10):608-24.

62. Zhou C, Molinie B, Daneshvar K, Pondick JV, Wang J, Van Wittenberghe N, et al. Genome-wide maps of m6A circRNAs identify widespread and celltype-specific methylation patterns that are distinct from mRNAs. Cell Rep. 2017;20(9):2262-76.

63. Zhang L, Hou C, Chen C, Guo Y, Yuan W, Yin D, et al. The role of $N^{6}$ methyladenosine $\left(\mathrm{m}^{6} \mathrm{~A}\right)$ modification in the regulation of circRNAs. Mol Cancer. 2020;19(1):105.

64. Yang $Y$, Fan $X$, Mao M, Song $X$, Wu $P$, Zhang $Y$, et al. Extensive translation of circular RNAs driven by N${ }^{6}$-methyladenosine. Cell Res. 2017;27(5):626-41.

65. Chen YG, Chen R, Ahmad S, Verma R, Kasturi SP, Amaya L, et al. N6Methyladenosine modification controls circular RNA immunity. Mol Cell. 2019;76(1):96-109.

66. Guo JU, Agarwal V, Guo H, Bartel DP. Expanded identification and characterization of mammalian circular RNAs. Genome Biol. 2014;15(7):409.
67. Chen CY, Sarnow P. Initiation of protein synthesis by the eukaryotic translational apparatus on circular RNAs. Science. 1995;268(5209):415-7.

68. Perriman R, Ares MJ. Circular mRNA can direct translation of extremely long repeating-sequence proteins in vivo. RNA. 1998:4(9):1047-54.

69. Pamudurti NR, Bartok O, Jens M, Ashwal-Fluss R, Stottmeister C, Ruhe L, et al. Translation of CircRNAs. Mol Cell. 2017;66(1):9-21.

70. Lei M, Zheng G, Ning Q, Zheng J, Dong D. Translation and functional roles of circular RNAs in human cancer. Mol Cancer. 2020;19(1):30.

71. Conn VM, Hugouvieux V, Nayak A, Conos SA, Capovilla G, Cildir G, et al. A circRNA from SEPALLATA3 regulates splicing of its cognate mRNA through R-loop formation. Nat Plants. 2017;3:17053.

72. Xu X, Zhang J, Tian Y, Gao Y, Dong X, Chen W, et al. CircRNA inhibits DNA damage repair by interacting with host gene. Mol Cancer. 2020;19(1):128.

73. Panda AC. Circular RNAs act as miRNA sponges. Adv Exp Med Biol. 2018; 1087:67-79.

74. Thomson DW, Dinger ME. Endogenous microRNA sponges: evidence and controversy. Nat Rev Genet. 2016;17(5):272-83.

75. Zhong Q, Huang J, Wei J, Wu R. Circular RNA CDR1as sponges miR-7-5p to enhance E2F3 stability and promote the growth of nasopharyngeal carcinoma. Cancer Cell Int. 2019;19:252.

76. Zou Y, Zheng S, Deng $X$, Yang A, Xie X, Tang H, et al. The Role of Circular RNA CDR1as/ciRS-7 in Regulating Tumor Microenvironment: A Pan-Cancer Analysis. Biomolecules. 2019:9(9):429.

77. Guo Z, Cao Q, Zhao Z, Song C. Biogenesis, features, functions, and disease relationships of a specific circular RNA: CDR1as. Aging Dis. 2020;11(4):1009-20.

78. Zhu P, Zhu X, Wu J, He L, Lu T, Wang Y, et al. IL-13 secreted by ILC2S promotes the self-renewal of intestinal stem cells through circular RNA circPan3. Nat Immunol. 2019;20(2):183-94.

79. Schuller AP, Green R. Roadblocks and resolutions in eukaryotic translation. Nat Rev Mol Cell Biol. 2018;19(8):526-41.

80. Jackson RJ, Hellen CU, Pestova TV. The mechanism of eukaryotic translation initiation and principles of its regulation. Nat Rev Mol Cell Biol. 2010;11(2): $113-27$

81. Zhao J, Lee EE, Kim J, Yang R, Chamseddin B, Ni C, et al. Transforming activity of an oncoprotein-encoding circular RNA from human papillomavirus. Nat Commun. 2019:10(1):2300.

82. Macejak DG, Sarnow P. Internal initiation of translation mediated by the $5^{\prime}$ leader of a cellular mRNA. Nature. 1991:353(6339):90-4

83. Legnini I, Di Timoteo G, Rossi F, Morlando M, Briganti F, Sthandier O, et al. Circ-ZNF609 is a circular RNA that can be translated and functions in Myogenesis. Mol Cell. 2017;66(1):22-37.

84. Abe N, Hiroshima M, Maruyama H, Nakashima Y, Nakano Y, Matsuda A, et al. Rolling circle amplification in a prokaryotic translation system using small circular RNA. Angew Chem Int Ed Eng. 2013;52(27):7004-8.

85. Yang Y, Gao X, Zhang M, Yan S, Sun C, Xiao F, et al. Novel role of FBXW7 circular RNA in repressing Glioma tumorigenesis. J Natl Cancer Inst. 2018; 110(3):304-15.

86. Pan Z, Cai J, Lin J, Zhou H, Peng J, Liang J, et al. A novel protein encoded by circFNDC3B inhibits tumor progression and EMT through regulating snail in colon cancer. Mol Cancer. 2020;19(1):71.

87. Huang A, Zheng H, Wu Z, Chen M, Huang Y. Circular RNA-protein interactions: functions, mechanisms, and identification. Theranostics. 2020;10(8):3503-17.

88. Luo J, Liu H, Luan S, Li Z. Guidance of circular RNAs to proteins' behavior as binding partners. Cell Mol Life Sci. 2019;76(21):4233-43.

89. Du WW, Zhang C, Yang W, Yong T, Awan FM, Yang BB. Identifying and characterizing circRNA-protein interaction. Theranostics. 2017;7(17):4183-91.

90. Schneider T, Hung LH, Schreiner S, Starke S, Eckhof H, Rossbach O, et al. CircRNA-protein complexes: IMP3 protein component defines subfamily of circRNPs. Sci Rep. 2016;6:31313.

91. You X, Vlatkovic I, Babic A, Will T, Epstein I, Tushev G, et al. Neural circular RNAs are derived from synaptic genes and regulated by development and plasticity. Nat Neurosci. 2015;18(4):603-10.

92. Du WW, Fang L, Yang W, Wu N, Awan FM, Yang Z, et al. Induction of tumor apoptosis through a circular RNA enhancing Foxo3 activity. Cell Death Differ. 2017;24(2):357-70.

93. Du WW, Yang W, Liu E, Yang Z, Dhaliwal P, Yang BB. Foxo3 circular RNA retards cell cycle progression via forming ternary complexes with p21 and CDK2. Nucleic Acids Res. 2016:44(6):2846-58.

94. Sun S, Wang W, Luo X, Li Y, Liu B, Li X, et al. Circular RNA circ-ADD3 inhibits hepatocellular carcinoma metastasis through facilitating EZH2 degradation via CDK1-mediated ubiquitination. Am J Cancer Res. 2019;9(8):1695-707. 
95. Zeng Y, Du WW, Wu Y, Yang Z, Awan FM, Li X, et al. A circular RNA binds to and activates AKT phosphorylation and nuclear localization reducing apoptosis and enhancing cardiac repair. Theranostics. 2017;7(16):3842-55.

96. Chen J, Zhou X, Yang J, Sun Q, Liu Y, Li N, et al. Circ-GLI1 promotes metastasis in melanoma through interacting with p70S6K2 to activate hedgehog/GLI1 and Wnt/beta-catenin pathways and upregulate Cyr61. Cell Death Dis. 2020;11(7):596.

97. Yang F, Fang E, Mei H, Chen Y, Li H, Li D, et al. Cis-acting circ-CTNNB1 promotes beta-catenin signaling and Cancer progression via DDX3mediated transactivation of YY1. Cancer Res. 2019:79(3):557-71.

98. Li H, Yang F, Hu A, Wang X, Fang E, Chen Y, et al. Therapeutic targeting of circ-CUX1/EWSR1/MAZ axis inhibits glycolysis and neuroblastoma progression. EMBO Mol Med. 2019;11(12):e10835.

99. Li Q, Wang Y, Wu S, Zhou Z, Ding X, Shi R, et al. CircACC1 regulates assembly and activation of AMPK complex under metabolic stress. Cell Metab. 2019;30(1):157-73.

100. Fang L, Du WW, Lyu J, Dong J, Zhang C, Yang W, et al. Enhanced breast cancer progression by mutant p53 is inhibited by the circular RNA circCcnb1. Cell Death Differ. 2018;25(12):2195-208.

101. Fang L, Du WW, Awan FM, Dong J, Yang BB. The circular RNA circ-Ccnb1 dissociates Ccnb1/Cdk1 complex suppressing cell invasion and tumorigenesis. Cancer Lett. 2019;459:216-26.

102. Yang F, Hu A, Li D, Wang J, Guo Y, Liu Y, et al. Circ-HuR suppresses HuR expression and gastric cancer progression by inhibiting CNBP transactivation. Mol Cancer. 2019;18(1):158.

103. Yang L, Han B, Zhang Z, Wang S, Bai Y, Zhang Y, et al. Extracellular VesicleMediated Delivery of Circular RNA SCMH1 Promotes Functional Recovery in Rodent and Nonhuman Primate Ischemic Stroke Models. Circulation. 2020; 142(6):556-74.

104. Pandey PR, Yang JH, Tsitsipatis D, Panda AC, Noh JH, Kim KM, et al. circSamd4 represses myogenic transcriptional activity of PUR proteins. Nucleic Acids Res. 2020;48(7):3789-805.

105. Zhou LY, Zhai M, Huang Y, Xu S, An T, Wang YH, et al. The circular RNA ACR attenuates myocardial ischemia/reperfusion injury by suppressing autophagy via modulation of the Pink1/ FAM65B pathway. Cell Death Differ. 2019;26(7):1299-315.

106. Xia P, Wang S, Ye B, Du Y, Li C, Xiong Z, et al. A circular RNA protects dormant hematopoietic stem cells from DNA sensor cGAS-mediated exhaustion. Immunity. 2018;48(4):688-701.

107. Barbagallo D, Caponnetto A, Cirnigliaro M, Brex D, Barbagallo C, D'Angeli F, et al. CircSMARCA5 Inhibits Migration of Glioblastoma Multiforme Cells by Regulating a Molecular Axis Involving Splicing Factors SRSF1/SRSF3/PTB. Int J Mol Sci. 2018;19(2):480

108. Barbagallo D, Caponnetto A, Brex D, Mirabella F, Barbagallo C, Lauretta G, et al. CircSMARCA5 Regulates VEGFA mRNA Splicing and Angiogenesis in Glioblastoma Multiforme Through the Binding of SRSF1. Cancers (Basel). 2019:11(2):194.

109. Abdelmohsen K, Panda AC, Munk R, Grammatikakis I, Dudekula DB, De S, et al. Identification of HuR target circular RNAs uncovers suppression of PABPN1 translation by CircPABPN1. RNA Biol. 2017;14(3):361-9.

110. Li XX, Xiao L, Chung HK, Ma XX, Liu X, Song JL, et al. Interaction between HuR and circPABPN1 Modulates Autophagy in the Intestinal Epithelium by Altering ATG16L1 Translation. Mol Cell Biol. 2020;40(6):e00492-19.

111. Zhu YJ, Zheng B, Luo GJ, Ma XK, Lu XY, Lin XM, et al. Circular RNAs negatively regulate cancer stem cells by physically binding FMRP against CCAR1 complex in hepatocellular carcinoma. Theranostics. 2019;9(12):3526-40.

112. Liu Y, Dong Y, Zhao L, Su L, Luo J. Circular RNAMTO1 suppresses breast cancer cell viability and reverses monastrol resistance through regulating the TRAF4/Eg5 axis. Int J Oncol. 2018;53(4):1752-62.

113. Xia B, Hong T, He X, Hu X, Gao Y. A circular RNA derived from MMP9 facilitates oral squamous cell carcinoma metastasis through regulation of MMP9 mRNA stability. Cell Transplant. 2019;28(12):1614-23.

114. Holdt LM, Stahringer A, Sass K, Pichler G, Kulak NA, Wilfert W, et al. Circular non-coding RNA ANRIL modulates ribosomal RNA maturation and atherosclerosis in humans. Nat Commun. 2016;7:12429.

115. Zhang C, Han X, Yang L, Fu J, Sun C, Huang S, et al. Circular RNA circPPM1F modulates M1 macrophage activation and pancreatic islet inflammation in type 1 diabetes mellitus. Theranostics. 2020;10(24):10908-24.

116. Hu X, Wu D, He X, Zhao H, He Z, Lin J, et al. circGSK3beta promotes metastasis in esophageal squamous cell carcinoma by augmenting betacatenin signaling. Mol Cancer. 2019;18(1):160.
117. Bi W, Huang J, Nie C, Liu B, He G, Han J, et al. CircRNA circRNA_102171 promotes papillary thyroid cancer progression through modulating CTNNBIP1-dependent activation of beta-catenin pathway. J Exp Clin Cancer Res. 2018;37(1):275.

118. Zhu Y, Gui W, Lin X, Li H. Knock-down of circular RNA H19 induces human adipose-derived stem cells adipogenic differentiation via a mechanism involving the polypyrimidine tract-binding protein 1. Exp Cell Res. 2020; 387(2):111753.

119. Shen S, Yao T, Xu Y, Zhang D, Fan S, Ma J. CircECE1 activates energy metabolism in osteosarcoma by stabilizing c-Myc. Mol Cancer. 2020;19(1): 151.

120. Zhang SB, Lin SY, Liu M, Liu CC, Ding HH, Sun Y, et al. CircAnks1a in the spinal cord regulates hypersensitivity in a rodent model of neuropathic pain. Nat Commun. 2019;10(1):4119.

121. Feng $Y$, Yang $Y$, Zhao $X$, Fan $Y$, Zhou L, Rong J, et al. Circular RNA circ0005276 promotes the proliferation and migration of prostate cancer cells by interacting with FUS to transcriptionally activate XIAP. Cell Death Dis. 2019;10(11):792.

122. Guarnerio J, Zhang Y, Cheloni G, Panella R, Mae KJ, Simpson M, et al. Intragenic antagonistic roles of protein and circRNA in tumorigenesis. Cell Res. 2019;29(8):628-40.

123. Chen N, Zhao G, Yan X, Lv Z, Yin H, Zhang S, et al. A novel FLI1 exonic circular RNA promotes metastasis in breast cancer by coordinately regulating TET1 and DNMT1. Genome Biol. 2018;19(1):218.

124. Jie M, Wu Y, Gao M, Li X, Liu C, Ouyang Q, et al. CircMRPS35 suppresses gastric cancer progression via recruiting KAT7 to govern histone modification. Mol Cancer. 2020;19(1):56.

125. Wang XH, Li J. CirCAGFG1 aggravates the progression of cervical cancer by downregulating p53. Eur Rev Med Pharmacol Sci. 2020;24(4):1704-11.

126. Zheng S, Qian Z, Jiang F, Ge D, Tang J, Chen H, et al. CircRNA LRP6 promotes the development of osteosarcoma via negatively regulating KLF2 and APC levels. Am J Transl Res. 2019;11(7):4126-38.

127. Ding $L$, Zhao Y, Dang S, Wang Y, Li X, Yu X, et al. Circular RNA circ-DONSON facilitates gastric cancer growth and invasion via NURF complex dependent activation of transcription factor SOX4. Mol Cancer. 2019;18(1):45.

128. Liu B, Ye B, Zhu X, Yang L, Li H, Liu N, et al. An inducible circular RNA circKcnt2 inhibits ILC3 activation to facilitate colitis resolution. Nat Commun. 2020;11(1):4076.

129. Hong Y, Qin H, Li Y, Zhang Y, Zhuang X, Liu L, et al. FNDC3B circular RNA promotes the migration and invasion of gastric cancer cells via the regulation of E-cadherin and CD44 expression. J Cell Physiol. 2019;234(11): 19895-910.

130. Chen L, Kong R, Wu C, Wang S, Liu Z, Liu S, et al. Circ-MALAT1 functions as both an mRNA translation brake and a microRNA sponge to promote selfrenewal of hepatocellular Cancer stem cells. Adv Sci (Weinh). 2020;7(4): 1900949.

131. Wu N, Yuan Z, Du KY, Fang L, Lyu J, Zhang C, et al. Translation of yesassociated protein (YAP) was antagonized by its circular RNA via suppressing the assembly of the translation initiation machinery. Cell Death Differ. 2019;26(12):2758-73.

132. Sun YM, Wang WT, Zeng ZC, Chen TQ, Han C, Pan Q, et al. circMYBL2, a circRNA from MYBL2, regulates FLT3 translation by recruiting PTBP1 to promote FLT3-ITD AML progression. Blood. 2019;134(18):1533-46.

133. Yang ZG, Awan FM, Du WW, Zeng Y, Lyu J, Wu D, et al. The circular RNA interacts with STAT3, increasing its nuclear translocation and wound repair by modulating Dnmt3a and miR-17 function. Mol Ther. 2017;25(9):2062-74.

134. Du WW, Yang W, Li X, Awan FM, Yang Z, Fang L, et al. A circular RNA circDNMT1 enhances breast cancer progression by activating autophagy. Oncogene. 2018;37(44):5829-42.

135. Zhao H, Chen S, Fu Q. Exosomes from CD133(+) cells carrying circ-ABCC1 mediate cell stemness and metastasis in colorectal cancer. J Cell Biochem. 2020;121(5-6):3286-97.

136. Wang L, Zheng C, Wu X, Zhang Y, Yan S, Ruan L, et al. Circ-SOX4 promotes non-small cell lung cancer progression by activating the Wnt/beta-catenin pathway. Mol Oncol. 2020.

137. Du WW, Yang W, Chen Y, Wu ZK, Foster FS, Yang Z, et al. Foxo3 circular RNA promotes cardiac senescence by modulating multiple factors associated with stress and senescence responses. Eur Heart J. 2017;38(18):1402-12.

138. Wang S, Zhang Y, Cai Q, Ma M, Jin LY, Weng M, et al. Circular RNA FOXP1 promotes tumor progression and Warburg effect in gallbladder cancer by regulating PKLR expression. Mol Cancer. 2019;18(1):145. 
139. Huang R, Zhang Y, Bai Y, Han B, Ju M, Chen B, et al. $N^{6}$-Methyladenosine modification of fatty acid amide hydrolase messenger RNA in circular RNA STAG1-regulated astrocyte dysfunction and depressive-like behaviors. Biol Psychiatry. 2020;88(5):392-404.

140. Liu B, Yang G, Wang X, Liu J, Lu Z, Wang Q, et al. CircBACH1 (hsa_circ_ 0061395) promotes hepatocellular carcinoma growth by regulating p27 repression via HuR. J Cell Physiol. 2020;235(10):6929-41.

141. Dou YQ, Kong P, Li CL, Sun HX, Li WW, YU Y, et al. Smooth muscle SIRT1 reprograms endothelial cells to suppress angiogenesis after ischemia. Theranostics. 2020;10(3):1197-212

142. Huang $X$, He M, Huang S, Lin R, Zhan M, Yang D, et al. Circular RNA circERBB2 promotes gallbladder cancer progression by regulating PA2G4dependent rDNA transcription. Mol Cancer. 2019;18(1):166.

143. Du WW, Yang W, Li X, Fang L, Wu N, Li F, et al. The circular RNA circSKA3 binds integrin beta1 to induce Invadopodium formation enhancing breast Cancer invasion. Mol Ther. 2020;28(5):1287-98.

144. Deng N, Lei D, Huang J, Yang Z, Fan C, Wang S. Circular RNA expression profiling identifies hsa_circ_0011460 as a novel molecule in severe preeclampsia. Pregnancy Hypertens. 2019;17:216-25.

145. Zhang X, Chen L, Xiao B, Liu H, Su Y. Circ_0075932 in adipocyte-derived exosomes induces inflammation and apoptosis in human dermal keratinocytes by directly binding with PUM2 and promoting PUM2mediated activation of AuroraA/NF-kappaB pathway. Biochem Biophys Res Commun. 2019;511(3):551-8.

146. Garikipati V, Verma SK, Cheng Z, Liang D, Truongcao MM, Cimini M, et al. Circular RNA CircFndc3b modulates cardiac repair after myocardial infarction via FUS/NEGF-A axis. Nat Commun. 2019;10(1):4317.

147. Wei Y, Zhang Y, Meng Q, Cui L, Xu C. Hypoxia-induced circular RNA has_ circRNA_403658 promotes bladder cancer cell growth through activation of LDHA. Am J Transl Res. 2019;11(11):6838-49.

148. Ma J, Fang L, Yang Q, Hibberd S, Du WW, Wu N, et al. Posttranscriptional regulation of AKT by circular RNA angiomotin- like 1 mediates chemoresistance against paclitaxel in breast cancer cells. Aging (Albany NY). 2019;11(23):11369-81.

149. Zhao W, Wang S, Qin T, Wang W. Circular RNA (circ-0075804) promotes the proliferation of retinoblastoma via combining heterogeneous nuclear ribonucleoprotein $\mathrm{K}$ (HNRNPK) to improve the stability of E2F transcription factor 3 E2F3. J Cell Biochem. 2020;121(7):3516-25.

150. Yang H, Li X, Meng Q, Sun H, Wu S, Hu W, et al. CircPTK2 (hsa_circ_ 0005273) as a novel therapeutic target for metastatic colorectal cancer. Mol Cancer. 2020;19(1):13.

151. Jia Y, Li X, Nan A, Zhang N, Chen L, Zhou H, et al. Circular RNA 406961 interacts with ILF2 to regulate $\mathrm{PM}_{2.5}$-induced inflammatory responses in human bronchial epithelial cells via activation of STAT3/JNK pathways. Environ Int. 2020;141:105755

152. Jia M, Li X, Jiang C, Wang K, Zuo T, He G, et al. Testis-enriched circular RNA circ-Bbs9 plays an important role in Leydig cell proliferation by regulating a CyclinD2-dependent pathway. Reprod Fertil Dev. 2020;32(4):355-62.

153. Zhou Z, Jiang R, Yang X, Guo H, Fang S, Zhang Y, et al. circRNA mediates silica-induced macrophage activation via HECTD1/ZC3H12A-dependent Ubiquitination. Theranostics. 2018:8(2):575-92.

154. Wong CH, Lou UK, Li Y, Chan SL, Tong JH, To KF, et al. CircFOXK2 promotes growth and metastasis of pancreatic ductal adenocarcinoma by Complexing with RNA-binding proteins and sponging MiR-942. Cancer Res. 2020;80(11):2138-49

155. Gan X, Zhu H, Jiang X, Obiegbusi SC, Yong M, Long X, et al. CircMUC16 promotes autophagy of epithelial ovarian cancer via interaction with ATG13 and miR-199a. Mol Cancer. 2020;19(1):45.

156. Qin M, Wei G, Sun X. Circ-UBR5: An exonic circular RNA and novel small nuclear RNA involved in RNA splicing. Biochem Biophys Res Commun. 2018; 503(2):1027-34.

157. Diling C, Yinrui G, Longkai Q, Xiaocui T, Yadi L, Xin Y, et al. Circular RNA NF1-419 enhances autophagy to ameliorate senile dementia by binding Dynamin-1 and adaptor protein 2 B1 in AD-like mice. Aging (Albany NY). 2019;11(24):12002-31.

158. Nan A, Chen L, Zhang N, Jia Y, Li X, Zhou H, et al. Circular RNA circNOL10 inhibits lung Cancer development by promoting SCLM1-mediated transcriptional regulation of the Humanin polypeptide family. Adv Sci (Weinh). 2019;6(2):1800654
159. Si X, Zheng H, Wei G, Li M, Li W, Wang H, et al. circRNA Hipk3 induces cardiac regeneration after myocardial infarction in mice by binding to Notch1 and miR-133a. Mol Ther Nucleic Acids. 2020;21:636-55.

160. Liu Z, Wang Q, Wang X, Xu Z, Wei X, Li J. Circular RNA cIARS regulates ferroptosis in HCC cells through interacting with RNA binding protein ALKBH5. Cell Death Discov. 2020;6:72.

161. Yang W, Du WW, Li X, Yee AJ, Yang BB. Foxo3 activity promoted by noncoding effects of circular RNA and Foxo3 pseudogene in the inhibition of tumor growth and angiogenesis. Oncogene. 2016;35(30):3919-31.

162. Chen Q, Sun L, Chen ZJ. Regulation and function of the CGAS-STING pathway of cytosolic DNA sensing. Nat Immunol. 2016;17(10):1142-9.

163. Cheung HC, Hai T, Zhu W, Baggerly KA, Tsavachidis S, Krahe R, et al. Splicing factors PTBP1 and PTBP2 promote proliferation and migration of glioma cell lines. Brain. 2009;132(Pt 8):2277-88.

164. Stadhouders R, Filion GJ, Graf T. Transcription factors and 3D genome conformation in cell-fate decisions. Nature. 2019;569(7756):345-54.

165. Tasdemir E, Chiara MM, Morselli E, Criollo A, D'Amelio M, Djavaheri-Mergny $M$, et al. A dual role of p53 in the control of autophagy. Autophagy. 2008; 4(6):810-4.

166. Blankman JL, Cravatt BF. Chemical probes of endocannabinoid metabolism. Pharmacol Rev. 2013;65(2):849-71.

167. Pelletier J, Thomas G, Volarevic S. Ribosome biogenesis in cancer: new players and therapeutic avenues. Nat Rev Cancer. 2018;18(1):51-63.

168. Nguyen LX, Lee $Y$, Urbani L, Utz PJ, Hamburger AW, Sunwoo JB, et al. Regulation of ribosomal RNA synthesis in T cells: requirement for GTP and Ebp1. Blood. 2015;125(16):2519-29.

\section{Publisher's Note}

Springer Nature remains neutral with regard to jurisdictional claims in published maps and institutional affiliations.

Ready to submit your research? Choose BMC and benefit from:

- fast, convenient online submission

- thorough peer review by experienced researchers in your field

- rapid publication on acceptance

- support for research data, including large and complex data types

- gold Open Access which fosters wider collaboration and increased citations

- maximum visibility for your research: over $100 \mathrm{M}$ website views per year

At $\mathrm{BMC}$, research is always in progress.

Learn more biomedcentral.com/submissions 